\title{
Article \\ Identification of miRNA-mRNA Regulatory Modules Involved in Lipid Metabolism and Seed Development in a Woody Oil Tree (Camellia oleifera)
}

\author{
Bo Wu ${ }^{1}$, Chengjiang Ruan ${ }^{1}, * \mathbb{C}$, Asad Hussain Shah ${ }^{2}$, Denghui $\mathrm{Li}^{3}{ }^{3} \mathrm{He} \mathrm{Li}^{1}$, Jian Ding ${ }^{1}$, Jingbin Li ${ }^{1}$ and Wei Du ${ }^{1}$ \\ 1 Key Laboratory of Biotechnology and Bioresources Utilization, Ministry of Education, Institute of Plant \\ Resources, Dalian Minzu University, Dalian 116600, China; wb512703926@163.com (B.W.); \\ lihe@dlnu.edu.cn (H.L.); dingjian@dlnu.edu.cn (J.D.); lijingbin@dlnu.edu.cn (J.L.); duwei@dlnu.edu.cn (W.D.) \\ 2 Department of Biotechnology, Faculty of Sciences, University of Kotli Azad Jammu and Kashmir, \\ Azad Jammu and Kashmir, Kotli 11100, Pakistan; syedasadhamdani@gmail.com \\ 3 Guizhou Wulingshan Youcha Technology Innovation Research Institute Co., Ltd., Tongren 554300, China; \\ lengfengtaolue@163.com \\ * Correspondence: ruan@dlnu.edu.cn; Tel.: +86-411-87652536
}

check for updates

Citation: Wu, B.; Ruan, C.; Shah, A.H.; Li, D.; Li, H.; Ding, J.; Li, J.; Du, W. Identification of miRNA-mRNA Regulatory Modules Involved in Lipid Metabolism and Seed Development in a Woody Oil Tree (Camellia oleifera). Cells 2022, 11, 71. https://doi.org/10.3390/cells11010071

Academic Editors: Marek Figlerowicz and Anna Philips

Received: 15 December 2021

Accepted: 24 December 2021

Published: 27 December 2021

Publisher's Note: MDPI stays neutral with regard to jurisdictional claims in published maps and institutional affiliations.

Copyright: (C) 2021 by the authors. Licensee MDPI, Basel, Switzerland. This article is an open access article distributed under the terms and conditions of the Creative Commons Attribution (CC BY) license (https:// creativecommons.org/licenses/by/ $4.0 /)$.

\begin{abstract}
Tea oil camellia (Camellia oleifera), an important woody oil tree, is a source of seed oil of high nutritional and medicinal value that is widely planted in southern China. However, there is no report on the identification of the miRNAs involved in lipid metabolism and seed development in the high- and low-oil cultivars of tea oil camellia. Thus, we explored the roles of miRNAs in the key periods of oil formation and accumulation in the seeds of tea oil camellia and identified miRNA-mRNA regulatory modules involved in lipid metabolism and seed development. Sixteen small RNA libraries for four development stages of seed oil biosynthesis in high- and low-oil cultivars were constructed. A total of 196 miRNAs, including 156 known miRNAs from 35 families, and 40 novel miRNAs were identified, and 55 significantly differentially expressed miRNAs were found, which included 34 upregulated miRNAs, and 21 downregulated miRNAs. An integrated analysis of the miRNA and mRNA transcriptome sequence data revealed that 10 miRNA-mRNA regulatory modules were related to lipid metabolism; for example, the regulatory modules of athmiR858b-MYB82/MYB3/MYB44 repressed seed oil biosynthesis, and a regulation module of csimiR166e-5p-S-ACP-DES6 was involved in the formation and accumulation of oleic acid. A total of 23 miRNA-mRNA regulatory modules were involved in the regulation of the seed size, such as the regulatory module of hpe-miR162a_L-2-ARF19, involved in early seed development. A total of 12 miRNA-mRNA regulatory modules regulating growth and development were identified, such as the regulatory modules of han-miR156a_L+1-SPL4/SBP2, promoting early seed development. The expression changes of six miRNAs and their target genes were validated using quantitative real-time PCR, and the targeting relationship of the cpa-miR393_R-1-AFB2 regulatory module was verified by luciferase assays. These data provide important theoretical values and a scientific basis for the genetic improvement of new cultivars of tea oil camellia in the future.
\end{abstract}

Keywords: Camellia oleifera; small RNA sequencing; miRNA-mRNA regulatory modules; lipid metabolism; seed development

\section{Introduction}

Tea oil camellia (Camellia oleifera Abel.) is an important woody oil tree in southern China and has a cultivation history of over two thousand years. Together with oil palm, olive, and coconut, tea oil camellia is well known as one of the four major woody oil trees in the world [1,2]. Tea oil camellia seeds have a high oil content of up to $40 \%$; are rich in unsaturated fatty acids (the oleic acid content accounts for over $75 \%$ of all the fatty acids in the oil of the seeds); exhibit an abundance of bioactive compounds, such as 
vitamins, camelliaside, and tea polyphenols; and are an important source of high-grade edible vegetable oil [3]. The oil extracted from tea oil camellia seeds is not easily oxidized, as it has high chemical stability and can be preserved for a long time; it can significantly contribute to the prevention of cardiovascular diseases and cancer and reduce cholesterol and blood lipids [4,5]. However, the seed yield of tea oil camellia is not stable, and the seed oil content varies greatly among different germplasms, which seriously restricts the sustainable development of the tea oil camellia industry. One important strategy is the breeding of tea oil camellia cultivars with high seed oil contents and high and stable seed yields.

MicroRNAs (miRNAs) are a class of endogenous noncoding small RNAs with lengths of approximately 20-25 nucleotides, and they play a crucial role in mediating the posttranscriptional regulation of gene expression by targeting mRNAs for degradation or inhibiting their translation in eukaryotes. MiRNAs are derived from primary miRNA transcripts (pri-miRNAs) that contain a stem-loop secondary structure; the pri-miRNA is processed in the nucleus by DCL1, a Dicer-like protein, to create an miRNA-miRNA* duplex, where miRNA* is a passenger strand complementary to the miRNA. The duplex is then separated by helicase, and the mature miRNA is incorporated into an ARGONAUTE 1 (AGO1) protein to form an RNA-induced silencing complex (RISC) [6,7]. Target genes that contain a sequence with almost complete complementarity to the miRNA are cleaved by the RISC at a specific site opposite to the 10th or 11th nucleotide in the miRNA [8]. Studies have confirmed the key roles of miRNAs in various biological and metabolic processes in plants, such as fatty acid biosynthesis [9,10], lipid metabolism [11,12], growth and development [13], responses to stresses [14], and cellular proliferation and differentiation [15]. As of 3 December 2021, a total of 38,589 miRNA sequences have been deposited in the database, miRBase [16,17], and many conserved and novel miRNAs have been identified in soybeans [18], Arabidopsis [19], Brassica napus [20], rice [21], peonies [22], and maize [23]. In walnuts, jre-miRn105, jre-miRn434, jre-miR477d, and jre-miR156a.2 are key miRNAs that regulate FA synthesis, and jre-miRn411 and jre-miR399a.1 are closely related to oil accumulation [10]. MiRNAs control and influence a variety of physiological processes by regulating target genes. For example, the $K A S$ and $K A R$ genes, targeted by miR159 and miR156, respectively, are important for lipid biosynthesis [24]. The FAD2 gene, targeted by miR159b and miR5026, regulates and influences FA biosynthesis [18,25]. Enhancing microRNA167A expression in the seeds decreased the alpha-linolenic-acid content and increased the seed size in Camelina sativa [26]. MiR172 plays a highly important role in seed development by modulating its target ARF2 gene [11].

Transcription factors play critical roles in regulating lipid biosynthesis [27], such as WRINKLED1 and LEAFY COTYLEDON, MYB, SPL, ARF, and AP2 [28], which have been identified as targets of the major miRNAs [29]. On the basis of the high-throughput small RNA and degradome analyses of soybean seeds 15 days after flowering, 55 annotated and 26 novel miRNAs, which targeted 145 genes, were identified [18]; a total of $82 \%$ of the targeted genes were transcription factors, including the ARF, MYB, TCP, GRF, and NAC families [18]. Known transcription-factor-encoding genes involved in seed size/weight determination, including SPL, GRF, MYB, ARF, HAIKU1, SHB1, KLUH/CYP78A5, and $E 2 F b$, along with novel genes, were found to be targeted by the predicted miRNAs in chickpeas [30]. MiRNA-target expression profiles evidenced that some miRNAs could tune distinct seed developmental stages by targeting the HD-ZIP, ARF, SPL, and NF-Y transcription-factor families in Phaseolus vulgaris [31]. MiR160 negatively regulates ARFs that significantly affect seed development in A. thaliana [32]. SPL10 and SPL11 are targeted by miR156 and are involved in the early morphogenesis of Arabidopsis embryos [33]. MYBs targeted by miR159 are involved in seed size, and a double mutation of miR159 (miR159ab) increased MYB33 and MYB65 expression, which promoted the formation of small seeds [34]. The transcription factor, WRI1, targeted by hrh-miRn215 in sea buckthorn seeds, involved in oil biosynthesis and fatty acid formation and accumulation [35], ethylene-responsive transcription factor 3 (ERF3), MADS domain protein, AGAMOUS-LIKE 61 (AGL61), and 
WRKY transcription factor 41 (WRKY41) were targeted by miR171k-5p_1, miR7760-p3_1, and Xso-miRn80 in the seeds of Xanthoceras sorbifolium [11].

Currently, miRNA-mRNA regulatory modules controlling multiple biological and metabolic processes have been revealed via the functional analysis of woody oil plants, such as hazel [36], oil palm [37], olive [38], peony [22], walnut [10], and yellowhorn [11]. In developing sea buckthorn seeds, 19 (4 known and 15 novel) and 22 (6 known and 16 novel) miRNAs were found to be involved in lipid biosynthesis and seed size, respectively [39], and an integrated analysis of the mRNA and miRNA transcriptomes and the qRT-PCR revealed key miRNAs and their targets (miR164d-ARF2, novelmiRNA-108-ACC, novelmiRNA-23-GPD1, novelmiRNA-58-DGAT1, and novelmiRNA-191-DGAT2) that are potentially involved in the seed size and lipid biosynthesis in the seeds of sea buckthorn. In developing seeds of yellowhorn, 141 differentially expressed miRNAs (120 known and 21 novel miRNAs) were identified, along with several miRNA-target regulatory modules involved in the lipid biosynthesis of yellowhorn seeds, such as miR319p_1-FAD2-2, miR5647-p3_1-DGAT1, and miR7760-p5_1-MED15A [11]. However, there are no reports on the roles of miRNAs in the different developmental stages of the seeds of high- and low-oil cultivars of tea oil camellia.

To understand the potential roles of miRNAs and their target genes in the developing seeds of tea oil camellia, high-throughput Illumina sequencing technology was used to reveal the global profile of small RNAs in the developing seeds of high- and low-oil tea oil camellia cultivars. Then, we identified some novel and known miRNAs and their target genes by the integrated analysis of the mRNA and miRNA transcriptomes. Finally, we further analyzed differentially expressed miRNAs and the GO and KEGG enrichments of these miRNAs target genes. In addition, reverse transcription-quantitative PCR (qRTqPCR) was used to validate the sequencing results for a selected group of miRNAs and the expression profiles of their targets, and the targeting relationship of the miRNA-mRNA regulatory module was verified by luciferase assays. Our datasets and results will promote the development of tea oil camellia miRNAs in public resource databases, and will provide a scientific resource and basis for further investigations of the identified miRNAs of tea oil camellia and their regulatory roles in the genetic improvement of high-quality and high-yielding tea oil camellia in the future.

\section{Materials and Methods}

\subsection{Study Site and Sampling}

The sample trees were from two cultivars of tea oil camellia, the cultivar, 'M3', with a high seed oil content, and the cultivar, 'M8', with a low seed oil content (voucher No. 1303 and No. 1308, respectively, identified by Chengjiang Ruan and deposited at Dalian Minzu University) growing in the tea oil camellia orchard in Yuping Dong Autonomics County, Guizhou Province, China. It is located at $27^{\circ} 28^{\prime}-27^{\circ} 31^{\prime}$ north latitude, and $108^{\circ} 34^{\prime}-109^{\circ} 09^{\prime}$ east longitude. The orchard had a mean annual rainfall of $1174.1 \mathrm{~mm}$, a mean annual temperature of $16.4{ }^{\circ} \mathrm{C}$, and a mean annual sunshine rate of 1227.8 . These two cultivars were selected from the tea oil camellia orchard because they had very similar phenotypic characteristics and genetic backgrounds, but not seed oil content.

For tea oil camellia, the key period of seed oil biosynthesis and accumulation is from June to September [40]. The oil contents in the seeds of these two cultivars showed a gradually upward trend from early June to early July; then, they began to rise rapidly from early July to early September and, subsequently, remained stable. The fruits were collected at random from the different plants of both cultivars on 2 June, 4 July, 5 August, and 3 September 2016, which were the same dates as those for the published mRNA-seq data [41], and the four sampling periods were referred to as T1 T4, for each cultivar in the data analysis; thus, we recorded them as M3T1, M3T2, M3T3, and M3T4, and as M8T1, M8T2, M8T3, and M8T4 for the samples of the two cultivars of the four periods, respectively. The seeds obtained by stripping the fruit shells were immediately wrapped in tin foil and placed into liquid nitrogen. The frozen seeds were transported to the laboratory and stored 
at $-80{ }^{\circ} \mathrm{C}$ for the following experiments. The collection of all the samples fully complied with local and national legislation.

\subsection{Small RNA Library Construction and Sequencing}

The total RNA of each sample was extracted by using TRIzol reagent (Invitrogen, Carlsbad, CA, USA), according to the manufacturer's instructions. The quality and quantity of the total RNA of each sample were analyzed using the Bioanalyzer 2100 (Agilent, Santa Clara, CA, USA) and RNA 6000 Nano LabChip Kit (Agilent, Santa Clara, CA, USA), ensuring that the RIN (RNA integrity number value) was $>7.0$. The small RNA library for each sample was constructed with the TruSeq Small RNA Sample Prep Kit (Illumina, San Diego, CA, USA), in accordance with the manufacturer's protocol. Then, we performed single-end sequencing ( $36 \mathrm{bp}$ or $50 \mathrm{bp}$ ) on an Illumina HiSeq2500 at LC-BIO (Hangzhou, China), following the vendor's recommended protocol.

\subsection{Identification and Analysis of miRNA Sequencing Data}

To obtain accurate and reliable miRNA sequencing data, the raw read quality was evaluated using Illumina FastQC to obtain Q30 data. Then, the raw reads were processed to obtain valid reads with an in-house program, ACGT101-miR (LC Sciences, Houston, TX, USA) [42], to filter out adapter-dimer reads, junk reads, low-quality reads, low-complexity reads, reads for common RNA families (rRNA, tRNA, snRNA, and snoRNA), and repeats. Then, the unique sequences, with lengths of $18-25 \mathrm{nt}$, were selected to map to the precursors of 66 specific species (Table S1) in miRBase 21.0 (http:/ / www.mirbase.org/, accessed on 3 August 2017)), through a BLAST search, to identify known miRNAs and novel 3pand 5p-derived miRNAs [43]. Because miRNAs are conserved, related species of tea oil camellia were selected as specific species. In the alignment analysis, the seed length was set to 16 , and the length variation at both the $3^{\prime}$ and $5^{\prime}$ ends, and one mismatch inside the sequence, were allowed. The unique sequences mapping to the hairpin arms of the mature miRNAs of specific species were identified as known miRNAs, and the unique sequences mapping to the other arms of known specific species' precursor hairpins opposite to the annotated mature miRNA-containing arms were considered to be novel $5 p$ - or $3 p-$ derived miRNA candidates [44]. The remaining sequences were mapped to other selected species precursors (with the exclusion of specific species) in miRBase 21.0 by a BLAST search, and the mapped pre-miRNAs were further BLAST-searched against the specific species genomes to determine their genomic locations. We defined the above two as known miRNAs. The unmapped sequences were BLAST-searched against specific genomes, and the hairpin RNA structures containing sequences were predicted from the flanking $120 \mathrm{nt}$ sequences using the RNAfold software (http:/ / rna.tbi.univie.ac.at/cgi-bin/RNAfold.cgi, accessed on 13 October 2017)). The criteria for the secondary structure prediction were: (1) The number of nucleotides in one bulge in the stem $(\leq 12)$; (2) The number of base pairs in the stem region of the predicted hairpin $(\geq 16) ;(3)$ The cutoff of free energy $(\mathrm{kCal} / \mathrm{mol}$ $\leq-15$ ); (4) The length of the hairpin (up and down stems + terminal loop $\geq 50$ ); (5) The length of the hairpin loop $(\leq 200) ;(6)$ The number of nucleotides in one bulge in the mature region $(\leq 4) ;(7)$ The number of bias errors in one bulge in the mature region $(\leq 2) ;(8)$ The number of biased bulges in the mature region $(\leq 2) ;(9)$ The number of errors in the mature region $(\leq 4) ;(10)$ The number of base pairs in the mature region of the predicted hairpin $(\geq 12)$; and (11) The percent of mature sequence in the stem $(\geq 80)$. After alignment analysis, the miRNAs that met the above criteria were considered novel miRNAs.

\subsection{Analysis of Differentially Expressed miRNAs}

The number of clean reads originating from each miRNA represents the relative expression abundance, or the level of the corresponding miRNA in small RNA deep sequencing. Normalized read counts were calculated for miRNA differential expression analysis according to the procedure in a previous study [45], with minor modifications: (1) Find a set of sequences common among all the samples; (2) Construct a reference dataset. 
Each datum in the reference set is the copy number median value of a corresponding common sequence of all the samples; (3) Perform base-2 logarithm transformation on the copy numbers $\left(\log _{2}(\operatorname{copy} \#)\right)$ of all the samples and reference the dataset; (4) Calculate the $\left(\log _{2}(\operatorname{copy} \#)\right)$ difference $\left(\Delta \log _{2}(\operatorname{copy} \#)\right)$ between the individual sample and the reference dataset; (5) Form a subset of sequences by selecting $\mid \Delta \log _{2}($ copy\#) $\mid<2$, which means less than a $\left(2^{2}=\right)$ four-fold change from the reference set; (6) Perform linear regressions between individual samples and the reference set on the subset sequences to derive linear equations, $\mathrm{y}=a_{\mathrm{i}} \mathrm{x}+b_{\mathrm{i}}$, where $a_{\mathrm{i}}$ and $b_{\mathrm{i}}$ are the slope and intercept, respectively, of the derived line; $x$ is the $\log _{2}$ (copy\#) of the reference set; and $y$ is the expected $\log _{2}$ (copy\#) of sample $\mathrm{i}$ on a corresponding sequence; (7) Calculate the mid value, $\mathrm{x}_{\operatorname{mid}}=(\max (\mathrm{x})-\min$ $(\mathrm{x})) / 2$ of the reference set. Calculate the corresponding expected $\log _{2}$ (copy\#) of sample $\mathrm{i}$, $\mathrm{y}_{\mathrm{i}, \mathrm{mid}}=a_{\mathrm{i}} \mathrm{x}_{\mathrm{mid}}+b_{\mathrm{i}}$. Let $\mathrm{y}_{\mathrm{r}, \text { mid }}=\mathrm{x}_{\mathrm{mid}}$, and let $\Delta \mathrm{y}_{\mathrm{i}}=\mathrm{y}_{\mathrm{r}, \text { mid }}-\mathrm{y}_{\mathrm{i}, \text { mid }}$, which is the logarithmic correction factor for sample $i$. We then derive the arithmetic correction factor, $f \mathrm{i}=2^{\Delta \mathrm{yi}}$, for sample $i$; (8) Correct the copy numbers for the individual samples by multiplying the corresponding arithmetic correction factor, $f \mathrm{i}$, by the original copy numbers.

A Student's $t$-test was used to identify miRNAs differentially expressed between two samples on the basis of the normalized read counts. An analysis of variance (ANOVA) was used to identify miRNAs differentially expressed among samples at the four development stages in each line by using the mirnaTA software [46]. The miRNAs with $p$-values $<0.05$ were considered differentially expressed miRNAs [45]. The normalized read counts of some miRNAs were set to be 0.01 for further calculation if they had no reads in the library.

\subsection{Prediction and Identification of miRNA-mRNA Regulatory Modules}

The target genes of differentially expressed miRNAs were identified by aligning mature miRNA sequences with published mRNA-seq sequences [41] using TargetFinder (https://github.com/carringtonlab/TargetFinder, accessed on 8 April 2018) [47], following the procedures described in previous studies [48]. The TargetFinder algorithm is based on the miRNA and mRNA complementary-pairing principle. The alignment score value in the prediction result was used as the screening standard, which was the score of the prediction result. The comparison between the miRNA and mRNA was performed, and then the complementary pairing score was given for the comparison position: a total of 1 point was given for one mismatch or deletion, and 0.5 points were given for one G:U pairing; the penalty for a mismatched G:U pairing in the core region was doubled, and the core region began at the 2 nd nt and continued to the 13th nt of the miRNA $\left(5^{\prime}-3^{\prime}\right)$. Finally, the results were obtained for scores less than or equal to 4 . The significance of the alignment score was the degree of match between the target gene and the miRNA. The lower the score, the more complete the matching, and the more credible it was.

The GO terms and KEGG pathways of genes targeted by these differentially expressed miRNAs were annotated. The GO function and KEGG information of this species were used to perform GO function annotation (http:/ / bioinfo.cau.edu.cn/agriGO/, accessed on 1 September 2018) and KEGG signaling pathway annotation (https:/ /www.kegg.jp/ $\mathrm{kegg} /$ pathway.html, accessed on 1 September 2018) for the target genes of differentially expressed miRNAs. Fisher's exact hypothesis test was performed on the GO and KEGG pathways of the targeted genes, and the enrichment analysis of each GO term and KEGG pathway was separately performed. According to the prediction results for the targeted genes of the differentially expressed miRNAs, significance differences in the GO and KEGG information were defined with a default threshold, $p<0.05$.

\subsection{Identification of the Expression of miRNAs and Their Target Genes by qRT-PCR}

To validate the high-throughput sequencing data, six pairs of miRNA target genes, on the basis of the sequencing data, were selected to perform the qRT-PCR. The total RNA from each sample for the qRT-PCR analysis was extracted, as described above, for the sequencing experiments. First-strand cDNA was synthesized using the Mir-X miRNAs First-Strand Synthesis Kit (TaKaRa Biotech, Dalian, China), according to the manufacturer's 
instructions. The U6 snRNA was selected as the reference gene, and the qRT-PCR for the miRNA experiments was performed in an ABI7500 real-time PCR instrument (Applied Biosystems, Foster City, CA, USA), using the Mir-X miRNA qRT-PCR SYBR Kit (TaKaRa Biotech, Dalian, China) and miRNA-specific primers (Table S2). The qRT-PCR experiments for each sample were performed with three replicates.

The predicted target genes of the miRNAs were selected for qRT-PCR analyses. Firststrand cDNA was synthesized using the PrimeScript ${ }^{\mathrm{TM}}$ RT Master Mix (TaKaRa Biotech, Dalian, China), according to the manufacturer's instructions. All the specific primer pairs of the predicted target genes (Table S3) were designed using the Primer Quest online software (http:/ / sg.idtdna.com/PrimerQuest/Home/Index, accessed on 10 September 2018). Elongation factor 1-alpha $(C o E F 1 \alpha)$ was selected as a reference gene. The qRT-PCR experiments were performed using the SYBR Premix Ex Taq ${ }^{\text {TM }}$ II Kit (Tli RNaseH Plus; TaKaRa Biotech, Dalian, China). The qRT-PCR was conducted using the ABI7500 real-time PCR instrument (Applied Biosystems, Foster, CA, USA). The qRT-PCR analyses for each sample were performed in triplicate. The relative expression levels of each gene were calculated using the $2^{-\Delta \Delta \mathrm{Ct}}$ method [49].

\subsection{Dual-Luciferase Reporter Assay}

Many studies have shown the AFB2 gene and miR393 to be involved in plant growth and development [50,51], but few have reported on the potential role of the miR393-AFB2 regulatory module in seeds. Thus, we investigated the targeting relationship between cpa-miR393_R-1 and AFB2 in the seeds of tea oil camellia. Fragments from the 3'-UTR of AFB2 containing the predicted binding sequences for cpa-miR393_R-1 were amplified and subcloned into the pmirGLO luciferase promoter vector. The pCDNA3.1 plasmid was used as the template vector. The fragment containing the nucleotide sequences of the precursor of cpa-miR393_R-1 was cloned into the vector to construct the recombinant vector expressing cpa-miR393_R-1 pCDNA3.1, as described previously. The pmirGLO vector containing the 3'-UTR of AFB2 was cotransfected with pCDNA3.1 or pCDNA3.1, containing pre-cpamiR393_R-1 into HEK-293T cells [52], using Lipofectamine 2000 (Invitrogen, Carlsbad, CA, USA), according to the manufacturer's protocol and a previous report [53-55]. Forty-eight hours after treatment, the firefly and Renilla luciferase activities were measured using a luciferase reporter assay kit (BioVision, Inc., Milpitas, CA, USA). Renilla was used as the transfection control.

\section{Results}

\subsection{Brief Outline for Sequencing Data, from Raw Data to Cleaned Sequences}

To comprehensively identify key small RNAs in the developing seeds of high- and low-oil cultivars of tea oil camellia, 16 small RNA libraries were sequenced on an Illumina HiSeq2500 platform for four different seed developmental stages, with two biological replicates for each seed developmental stage of each cultivar. To evaluate the consistency of the biological replicates, the Pearson correlation between the samples was calculated and is shown in Figure S1. A high correlation coefficient $(r)$ between every two replicates confirmed the reliability, as well as the operational stability, of the experimental results. Totals of 13,572,633 and 14,183,198 raw reads were obtained from the cultivars, 'M3' and 'M8', of tea oil camellia, respectively. Furthermore, the raw reads were analyzed with the LC sRNA analysis pipeline, ACGT101-miR, and totals of 11,653,829 (85.67\%) and 12,248,627 (86.23\%) valid reads were obtained for ' $\mathrm{M} 3$ ' and ' $\mathrm{M} 8$ ' after removing the adaptor dimers, junk reads, low-complexity sequences, and sequences shorter than 18 nucleotides, or longer than 25 nucleotides, respectively (Tables S4 and S5). The length distributions of the unique sRNAs for both cultivars at four developmental stages were then summarized. Most of the sRNA reads ranged from 21 to $24 \mathrm{nt}$ in length, leading to similar length distributions in two tea oil camellia cultivars at the different developmental stages. sRNAs of $24 \mathrm{nt}$ were the most abundant, accounting for $71.58 \%$ (M8T1) to $79.74 \%$ (M3T4) of the total (Figure 1). 
In addition, 21-, 22-, 23-, and 25-nt sRNAs were common, which were more abundant than those of any other length, besides $24 \mathrm{nt}$.

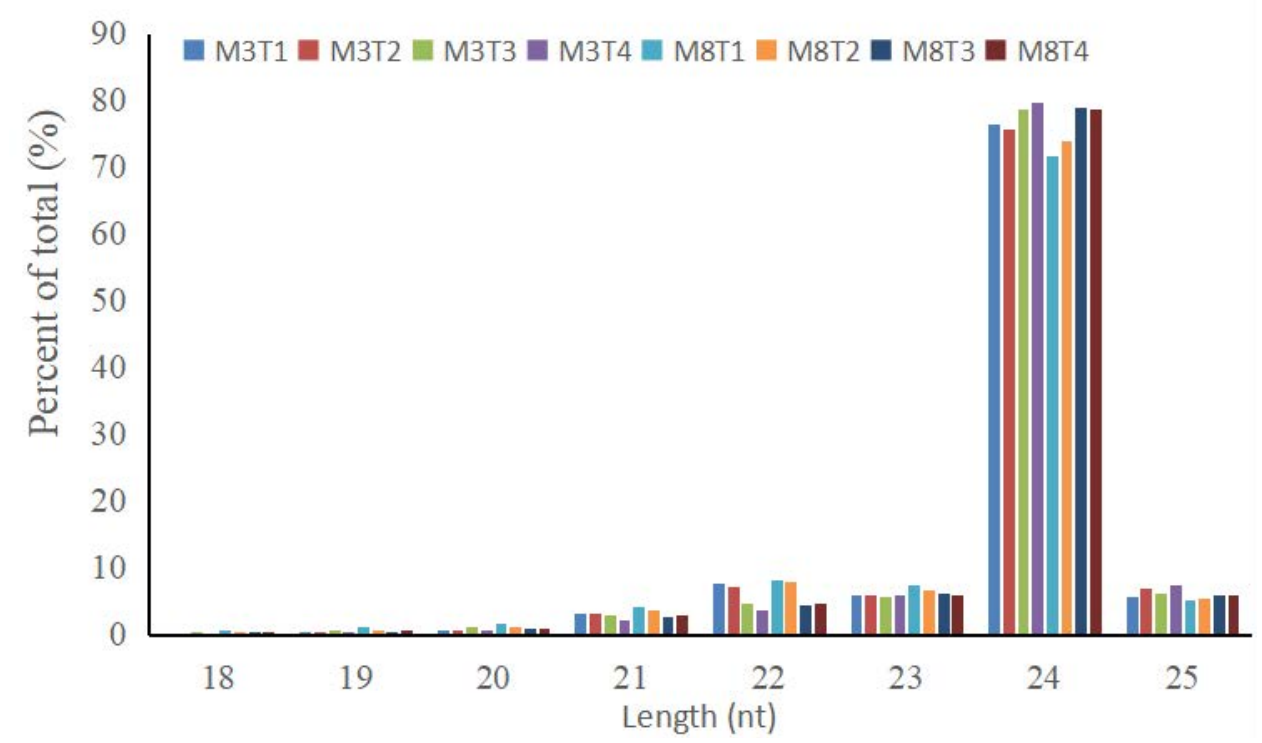

Figure 1. Length distributions of unique miRNAs from the seeds of the 'M3' and 'M8' cultivars of tea oil camellia at four different developmental stages.

\subsection{Identification of Known and Novel miRNAs in Developing Seeds of Tea Oil Camelia}

To detect the miRNAs involved in the development of tea oil camellia seeds, the unique sequences were selected and mapped against miRBase 21.0 by a BLAST search to identify the known miRNAs and novel miRNAs, and then a total of 196 expressed miRNAs were identified in the developing seeds of tea oil camellia (Table S6). The expressed miRNAs were classified into five groups (gp1, gp2a, gp2b, gp3, and gp4), according to criteria defined in a previous study [44], with minor modifications: seven known miRNAs belonged to gp1; 149 conserved and known miRNAs belonged to gp2a, gp2b, and gp3; and 40 novel miRNAs belonged to gp4 (Table S6). Analyses of the number and length ratios of unique miRNA sequences of different lengths revealed that the lengths of the miRNAs were mainly distributed within 20 24 nt (Table S7). MiRNAs with a length of $21 \mathrm{nt}$ were frequent, and accounted for $49.55 \%$ of all the miRNAs, which is consistent with the definition of miRNAs (Table S7). The results of the statistical analysis of the expression levels of the detected miRNAs, and the detection rates for miRNAs upon evaluating redundant miRNAs, showed that 196 unique miRNAs were identified, including 156 known miRNAs (Table S8), and 40 novel miRNAs (Table S9).

The miRNA sequences were mapped to known miRNAs from 42 plant species, with the highest number of miRNAs mapped to known gma-miRNAs of Glycine max (17 $(10.89 \%))$, followed by ath-miRNAs of Arabidopsis thaliana (13 (8.33\%)), and aly-miRNAs of Arabidopsis lyrata (11 (7.05\%)) (Table S8). Among the identified known miRNA sequences, 156 were identified as belonging to 35 miRNA families, and the MIR171 family had the largest number of members (12), followed by the MIR167 (11), and the MIR156 and MIR5645 families, with 10 members each (Table S8). The analysis of the distribution of the miRNA first-nucleotide preferences showed that miRNAs of $24 \mathrm{nt}$ tended to begin with $5^{\prime}-\mathrm{A}$ in tea oil camellia, while 21-22-nt miRNAs tended to start with $5^{\prime}-\mathrm{U}$, and 23-nt and 25-nt miRNAs tended to start with $5^{\prime}-\mathrm{G}$ (Figure $2 \mathrm{a}$ ). At the $5^{\prime}$ end, uridine (26.12\%) and adenine $(26.06 \%)$ were the similar common nucleotides for all the known miRNAs in tea oil camellia (Figure 2b). The expression profiles of the known miRNAs showed that the normalized read counts of the known miRNAs varied from 118,375 to less than 10 reads, exhibiting great variation, even within the same family. The normalized expression data were sorted from low to high. If the copy number for an identified miRNA was less than 10 in all the 
samples, or higher than 10 in one sample and less than the average copy number of the dataset in all the samples, this indicated that it had low expression; if the copy number of an identified miRNA was higher than the average copy number of the dataset in any sample, this indicated that it was highly expressed. According to the above classification method, 38 known miRNAs were highly expressed, and all the novel miRNAs had low expression. A total of 4 of the 40 miRNAs with low expression had copy numbers less than 10 in all the samples: col-miRn-5p-236956_11; col-miRn-3p-145806_25; col-miRn-3p-253727_10; and col-miRn-3p-150080_24. Compared to the known miRNAs, most of the identified novel miRNAs had relatively low read counts (normalized), and only five novel miRNAs had more than 100 reads in all the libraries: col-miRn-5p-1103_1978; col-miRn-5p-2142_1228; col-miRn-3p-18781_243; col-miRn-3p-3803_812; and col-miRn-3p-18137_250. The secondary structures of these five most abundant novel miRNAs were predicted (Figure S2), indicating that they can form typical stem-loop hairpins, and that their folding free energies were $-62.4,-71.9,-71.9,-50.0$, and $-66.9 \mathrm{kcal} \cdot \mathrm{mol}^{-1}$, respectively.
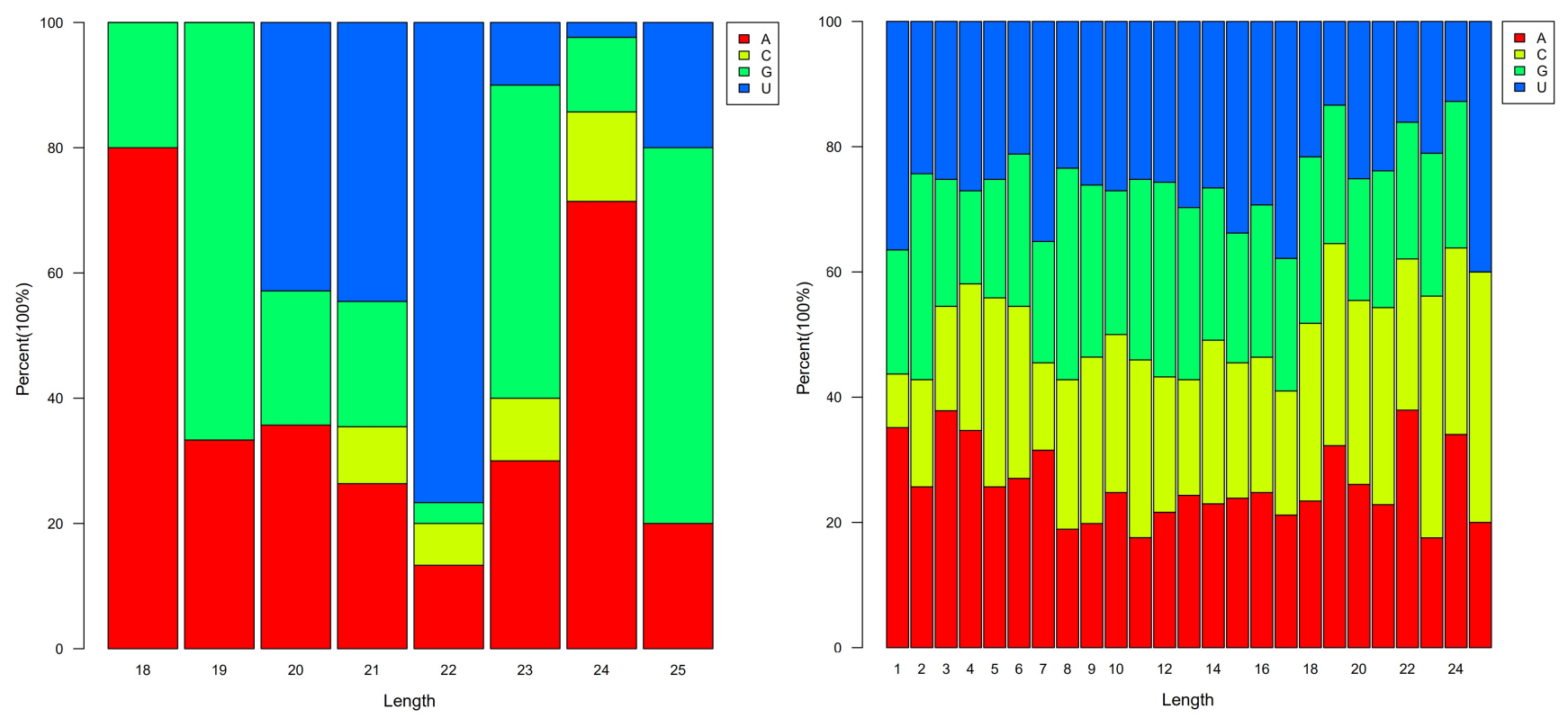

Figure 2. (a) First nucleotide, and (b) total nucleotide biases of tea oil camellia miRNA.

In addition, we detected the number of specifically expressed miRNAs in one sample, and the number of specifically expressed miRNAs simultaneously detected in two or more samples, which were visualized using a Venn diagram, comparing the samples of the two cultivars of 'M3'and 'M8' at four different developmental stages (Figure 3). It was found that 54 miRNAs were identified at four different seed developmental stages of the 'M3' cultivar (Figure 3a), and 63 miRNAs were identified for the 'M8' cultivar (Figure 3b). Mining these simultaneously expressed miRNAs could provide a favorable foundation for the genetic improvement of tea oil camellia.

\subsection{Identification of Differentially Expressed miRNAs}

The differentially expressed miRNAs were identified using suitable differential test methods, in which significantly differentially expressed miRNAs were defined with a significance threshold of a $p$-value $<0.05$. There were 55 significantly differentially expressed miRNAs identified between the samples of each cultivar at two different developmental stages, and between different samples of two cultivars at the same developmental stage. A total of 34 upregulated miRNAs, and 21 downregulated miRNAs, were identified among these significantly differentially expressed miRNAs (Figure S3). Moreover, to identify the expression level differences of the miRNAs significantly differentially expressed between the high- and low-oil cultivars of tea oil camelia, the significantly differentially expressed miRNAs were 
compared between the seeds of the cultivars of 'M3' and 'M8' at the same development stage (Figure 4, Table S10). At the early seed developmental stage, five miRNAs (mtrMIR2586a-p3_1ss15TC; ath-MIR5645b-p5_2ss19TG21TC; ath-MIR5645b-p3_2ss19TG21TC; mtr-MIR2586a-p3_2ss4TG21CT; and hpe-miR166a_1ss5AC) were upregulated in 'M3' relative to 'M8', and hpe-miR166a_1ss5AC had a 0.94-fold change (log2(ratio)). At the second seed developmental stage, three miRNAs (csi-miR1515_1ss16AG; stu-miR8016_1ss7GT; and ppe-miR172a-5p_1ss21GA) were upregulated in 'M3' relative to 'M8', with 0.38-, 1.86-, and 2.57-fold changes ( $\log 2$ (ratio)), respectively. Seven miRNAs (rco-miR156e_L+1R-1; dprmiR167a_R+1; ccl-miR167a,tcc-miR390a; ath-miR390a-5p; aly-miR167b-3p_1ss13AG; and han-miR156a_L+1) were downregulated, with -3.40-, -1.88-, -1.66-, -1.40-, -1.40-, -1.35-, and -2.81-fold changes ( $\log 2($ ratio) ), respectively. At the late seed developmental stage, four miRNAs (col-miRn-3p-9080_426; mtr-MIR2586a-p3_1ss15TC; ghr-MIR7499-p3_2ss10GC17CG; and rco-miR167a_R+1, with -0.33-fold changes (log2(ratio)), were downregulated in 'M3' compared with their expression in 'M8'.

(a)

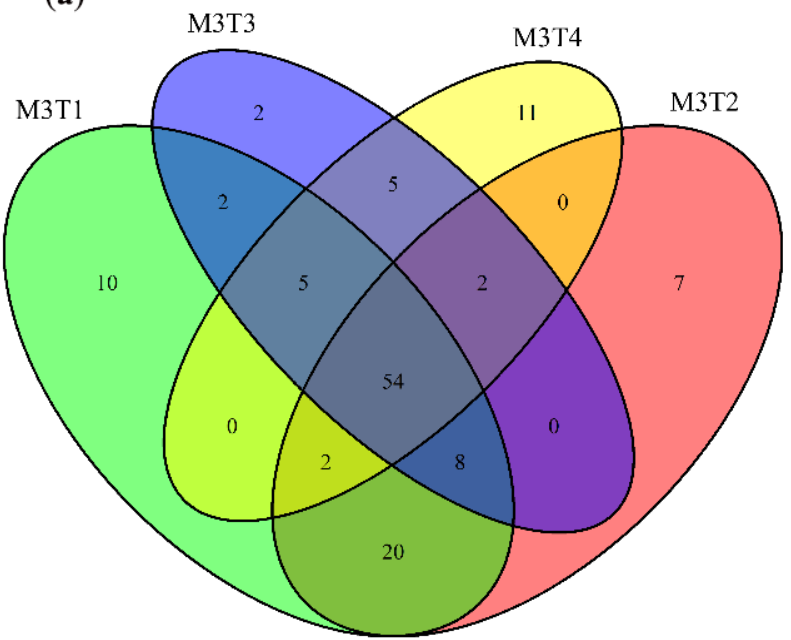

(b)

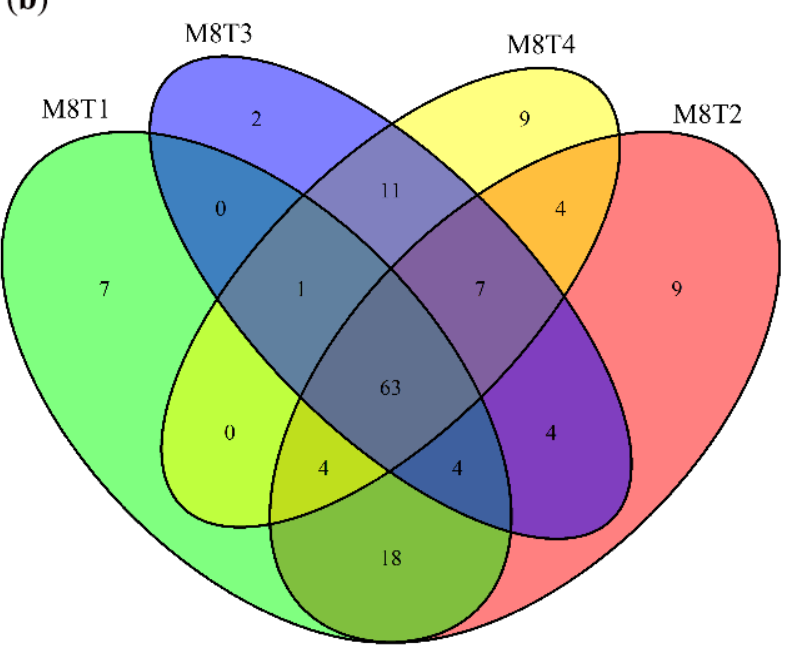

Figure 3. Venn diagrams of the detected miRNAs among (a) the seeds of 'M3', and (b) the seeds of 'M8', at four different developmental stages.

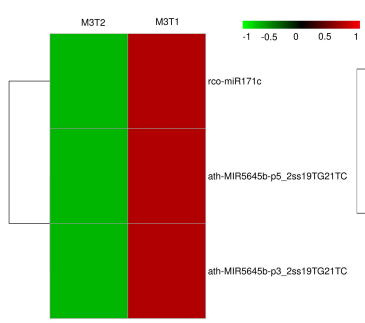

${ }_{\text {MงT4 }}$

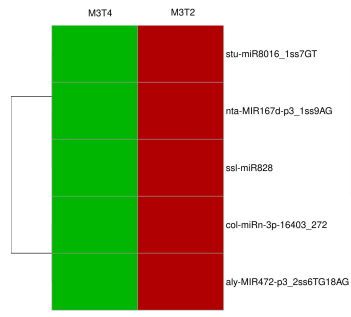

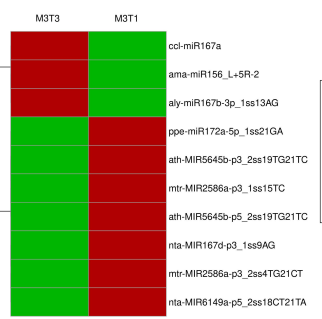

мвт2 мзг

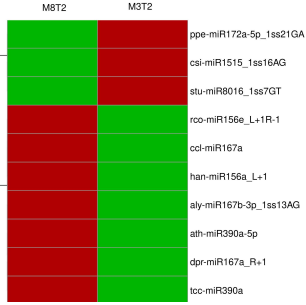

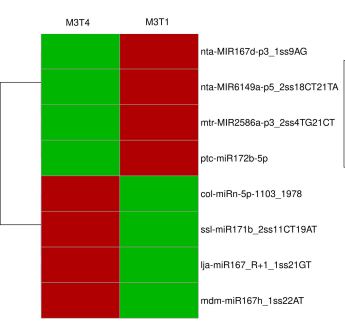

rcomilitita R.

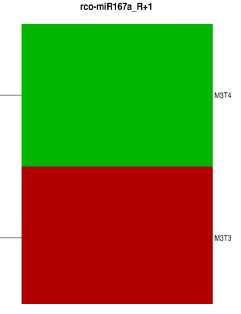

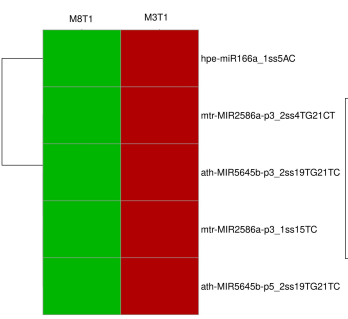

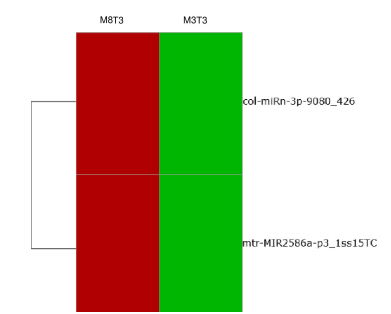

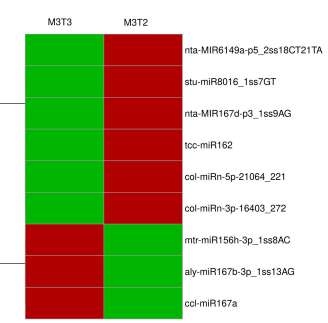

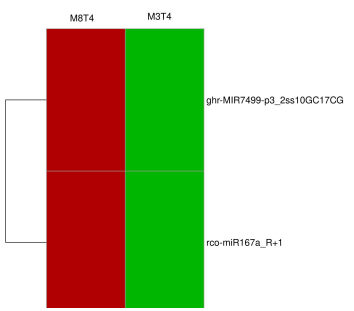

Figure 4. Heatmap of differentially expressed miRNAs among different groups.

In addition, the number of common and specific differentially expressed miRNAs is visually shown in a Venn diagram comparison between different comparison groups (Figure 5). The results show that there were four significantly differentially expressed miR- 
NAs (csi-miR1515_1ss16AG; ccl-miR167a; aly-miR167b-3p_1ss13AG; and ppe-miR172a5p_1ss21GA) simultaneously found in different comparison groups, when the significantly differentially miRNAs among different development stages in the ' $M 3$ ' seeds were compared with those of the comparison of M3T2 vs. M8T2 (Figure 5a). There were six significantly differentially expressed miRNAs (csi-miR1515_1ss16AG; ccl-miR167a; aly-miR167b-3p_1ss13AG; ppe-miR172a-5p_1ss21GA; aly-miR167b-3p_1ss13AG; and ppemiR172a-5p_1ss21GA) simultaneously found in different comparison groups, when the significantly differentially miRNAs among different development stages in the 'M8' seeds were compared with those of the comparison of M3T2 vs. M8T2 (Figure 5b). Moreover, six significantly differentially expressed miRNAs (aly-miR167b-3p_1ss13AG; ptc-miR172b-5p; ssl-miR828; csi-miR1515_1ss16AG; ath-miR858b; and ppe-miR172a-5p_1ss21GA) were simultaneously found in the comparison of the whole ' $\mathrm{M} 3$ ' and 'M8' seed developmental groups (Figure 5c), but only one differentially expressed miRNA (rco-miR167a_R+1) was simultaneously found between the comparison of the whole 'M3' seed developmental group and the comparison of M3T4 vs. M8T4 (Figure 5d). Mining for these significantly differentially expressed and simultaneous miRNAs is helpful for exploring the expression differences and regulatory patterns between the seeds of high- and low-oil cultivars of tea oil camellia at different developmental stages, which could provide a scientific basis for improving the seed oil content and quality of tea oil camellia in the future. These results suggest that miRNA-mediated regulatory mechanisms may play an important role in various biological pathways during seed development in tea oil camellia.
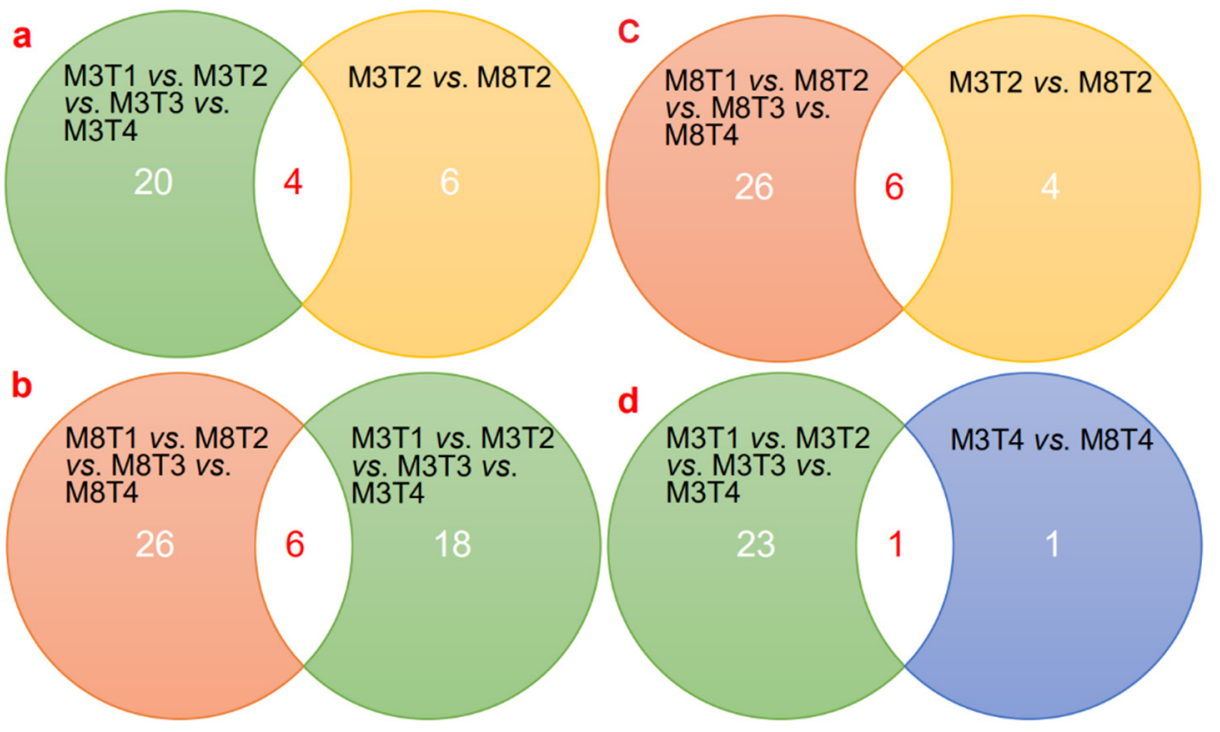

Figure 5. Venn diagrams of differentially expressed miRNAs between different comparison groups. (a) Distribution of a Venn diagram of differentially expressed miRNAs in M3T1 vs. M3T2 vs. M3T3 vs. M4T4 compared to M3T2 vs. M8T2, and M8T1 vs. M8T2 vs. M8T3 vs. M8T4 compared to M3T2 vs. M8T4; (b) Distribution of a Venn diagram of differentially expressed miRNAs in M3T1 vs. M3T2 vs. M3T3 vs. M4T4 compared to M8T1 vs. M8T2 vs. M8T3 vs. M8T4; (c) Distribution of a Venn diagram of differentially expressed miRNAs in M8T1 vs. M8T2 vs. M8T3 vs. M8T4 compared to M3T2 vs. M8T2; and (d) Distribution of a Venn diagram of differentially expressed miRNAs in M3T1 vs. M3T2 vs. M3T3 vs. M4T4 compared to M3T4 vs. M8T4.

\subsection{Prediction and Identification of miRNA-mRNA Regulatory Modules}

To further understand the functions of miRNAs in the developing seeds of high- and low-oil cultivars of tea oil camellia, the target genes of the differentially expressed miRNAs were predicted using the TargetFinder software on the basis of the miRNA sequences with published mRNA transcriptome data [41]. A total of 17,166 target genes were predicted, and some genes were targeted by multiple miRNAs, resulting in a total of 33,418 predicted 
miRNA-mRNA regulatory modules (Table S11). Furthermore, the predicted target genes of known and novel miRNAs were subjected to gene ontology (GO) analysis. The results show that the target genes were classified into three categories by the hypergeometric distribution algorithm (Figure 6a): biological process, cellular component, and molecular function. A total of 25,988 miRNA-mRNA regulatory modules were related to various biological processes, 21,132 of which were found to be involved in different cellular components, and 14,675 of which were identified to be involved in molecular function (Figure 6a). The major biological functions of differentially expressed target genes were identified by the significant terms in the GO enrichment analysis; these results show that a total of 107 significant GO terms were related to the nucleus, sequence-specific DNA binding, and the regulation of transcription, the DNA-template, and electron carrier activity (Figure $6 \mathrm{~b}$ and Table S12).
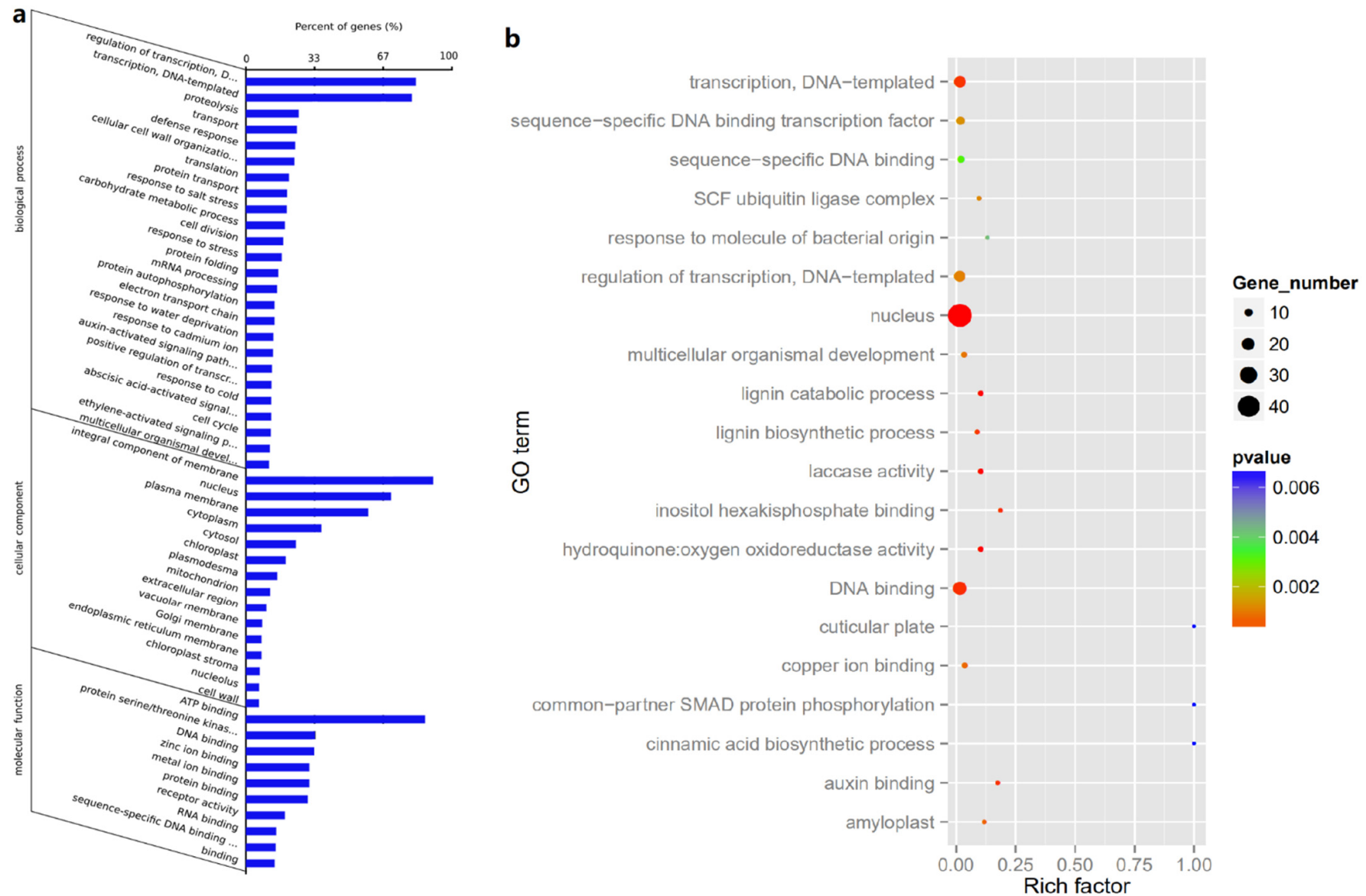

Figure 6. (a) GO categories and distribution, and (b) scatterplot distribution map of genes targeted by identified miRNAs in the seeds of tea oil camellia.

To further understand the biological functions of the target genes in the developing seeds of tea oil camellia, the target Kyoto Encyclopedia of Genes and Genomes (KEGG) pathway genes were identified by KEGG pathway enrichment analysis. These results show that a total of 16,871 miRNA-mRNA regulatory modules were involved in 206 individual KEGG pathways, involved in linoleic acid metabolism, alpha-linolenic acid metabolism, and glycosylated biosynthesis. The significant KEGG pathway enrichment analysis showed that 31 significant KEGG pathways (Figure 7 and Table S13) were mainly involved in pyruvate metabolism, the regulation of the actin cytoskeleton, fatty acid biosynthesis, and the citrate cycle. 


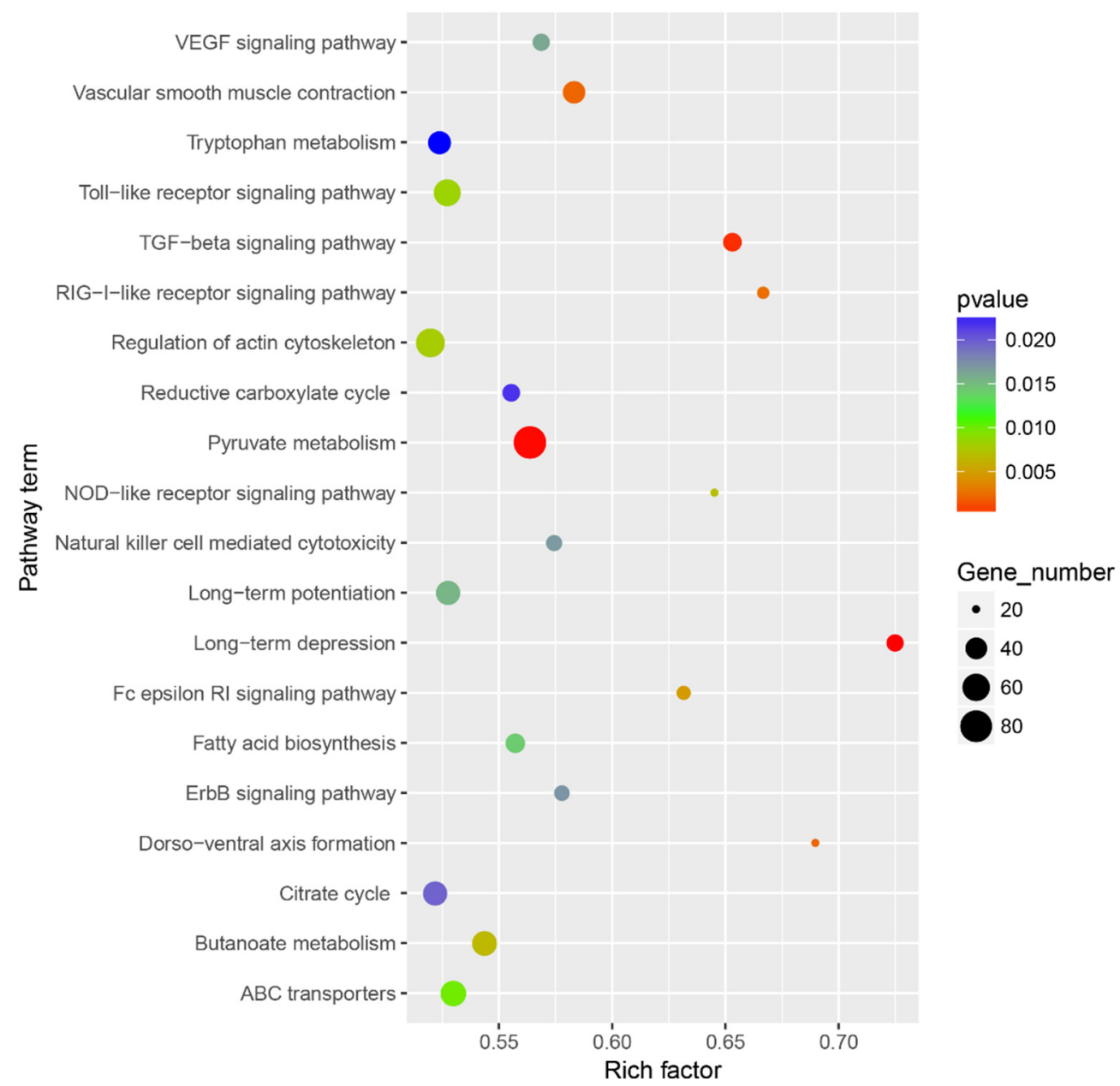

Figure 7. Analyses of significant KEGG enrichment scatterplot distribution map based on miRNA targets.

\subsection{MiRNA-mRNA Regulation Modules Involved in Lipid Metabolism}

The analyses of the differentially expressed miRNAs and their target genes involved in lipid metabolism revealed that multiple KEGG pathways were related to lipid metabolism during the seed development of tea oil camellia, according to the KEGG enrichment analysis $(p<0.05)$, in which 1869 miRNA-mRNA regulatory modules were involved in 12 pathways related to lipid metabolism, including 1723 predicted known miRNA-mRNA regulatory modules, and 146 novel miRNA-mRNA regulatory modules. In these regulation modules, the same miRNA often targeted different genes, and several miRNAs also targeted the same gene. There were 188 target genes involved in the fatty acid biosynthesis pathway, 411 target genes involved in the glycolysis/gluconeogenesis pathway, 118 target genes involved in the biosynthesis of the unsaturated fatty acid pathway, and 159 targeted genes related to the fatty acid metabolism pathway (Table S14). The significant KEGG enrichment analysis and function annotation were integrated, and five significant KEGG pathways related to lipid metabolism were identified, including pyruvate metabolism, fatty acid biosynthesis, glycolysis/gluconeogenesis, sphingolipid metabolism, and steroid biosynthesis.

A total of ten key miRNA-mRNA regulatory modules related to the lipid metabolism pathway were identified, including one novel miRNA (col-miRn-5p-21064_221), and eight known miRNAs that were involved in developing seeds of high- and low-oil cultivars of tea oil camellia (Table 1). These target genes were commonly involved in the seed oil biosynthesis pathway (Table S15). In the fatty acid biosynthesis metabolic pathway (ko00061), the ACC1 (comp59939_c1) gene was targeted by tcc-miR162. In the pyruvate metabolism metabolic pathway (ko00620), the KAS1 (comp67779_c0) and S-ACP-DES6 (comp67006_c0) 
genes were targeted by csi-miR166e-5p; the KAS3B (comp61049_c0) gene was targeted by byrgl-miR5139_L-1; the fabG (comp63911_c0) gene was targeted by mtr-miR156h3p_1ss8AC; the Mcat (comp63026_c1) gene was targeted by cpa-miR164d; the FATB1 (comp67050_c0) gene was targeted by col-miRn-5p-21064_221; the MOD1 (comp52017_c0) gene was targeted by aly-miR393a-3p_1ss12TC; and the SAD (comp48800_c0) gene was targeted by ath-miR172a. The gma-MIR5368-p3_1ss21CT targeted the GAPN (comp67185_c0) gene, involved in the glycolysis/gluconeogenesis metabolic pathway (ko00010).

Table 1. Predicted miRNAs and their target genes involved in lipid metabolism.

\begin{tabular}{|c|c|c|c|c|}
\hline miRNA ID & Gene ID & Gene Name & Gene Annotation & KEGG Pathway \\
\hline tcc-miR162 & comp59939_c1 & $A C C 1$ & $\begin{array}{l}\text { acetyl-CoA carboxylase/ } \\
\text { biotin carboxylase }\end{array}$ & $\begin{array}{l}\text { Pyruvate } \\
\text { metabolism }\end{array}$ \\
\hline csi-miR166e-5p & comp67779_c0 & KAS1 & 3-oxoacyl-[acyl-carrier-protein] synthase I & \\
\hline csi-miR166e-5p & comp67006_c0 & $S-A C P-D E S 6$ & acyl-[acyl-carrier-protein]-desaturase & \\
\hline rgl-miR5139_L-1 & comp61049_c0 & $K A S 3 B$ & $\begin{array}{c}\text { 3-oxoacyl-[acyl-carrier-protein] } \\
\text { synthase III }\end{array}$ & \\
\hline $\begin{array}{l}\text { mtr-miR156h- } \\
\text { 3p_1ss8AC }\end{array}$ & comp63911_c0 & $f a b G$ & 3-oxoacyl-[acyl-carrier protein] reductase & \\
\hline cpa-miR164d & comp63026_c1 & Mcat & $\begin{array}{l}\text { [acyl-carrier-protein] } \\
\text { S-malonyltransferase }\end{array}$ & $\begin{array}{l}\text { Fatty acid } \\
\text { biosynthesis } \\
\text { metabolic }\end{array}$ \\
\hline col-miRn-5p-21064_221 & comp67050_c0 & FATB1 & fatty acyl-ACP thioesterase B & \\
\hline $\begin{array}{l}\text { aly-miR393a- } \\
\text { 3p_1ss12TC }\end{array}$ & comp52017_c0 & MOD1 & enoyl-[acyl-carrier protein] reductase I & \\
\hline ath-miR172a & comp48800_c0 & $\Delta 9 D$ & acyl-[acyl-carrier-protein] desaturase & \\
\hline $\begin{array}{l}\text { gma-MIR5368- } \\
\text { p3_1ss21CT }\end{array}$ & comp67185_c0 & $G A P N$ & $\begin{array}{l}\text { glyceraldehyde-3-phosphate } \\
\text { dehydrogenase (NADP) }\end{array}$ & $\begin{array}{l}\text { Glycolysis / } \\
\text { gluconeogenesis }\end{array}$ \\
\hline
\end{tabular}

The expression patterns and expression differences of the above identified miRNAs and their target genes related to lipid metabolism were determined by the heat map analysis of their expression levels in the developing seeds of high- and low-oil cultivars of tea oil camellia (Figure 8). The heat map of the miRNAs and their target genes was produced using the MEV software, ver 4.9.0., on the basis of the original expression levels of the miRNAs, and their target genes were normalized by z-score normalization. On the one hand, at the developing seed levels of the 'M3' cultivar, the expression of cpa-miR164d was the highest at the early seed development stage, and it then showed a declining trend; however, the expression of its target gene, Mcat (comp63026_c1), was the lowest at this stage, and it then gradually increased. These results indicate that there may be a negative correlation between cpa-miR164d and the expression of its target gene, Mcat. The expression of mtr-miR156h-3p_1ss8AC was significantly downregulated in this stage, and then gradually increased; however, its target gene, fabG (comp63911_c0), was the highest, and it then gradually decreased to the lowest expression level in the late developmental stage. In addition, from the second to third seed developmental stages of the ' $\mathrm{M} 3$ ' cultivar, the expression of four miRNAs (aly-miR393a-3p_1ss12TC; rgl-miR5139_L-1; col-miRn-5p21064_221; and ath-miR172a) decreased gradually, while the expression of their target genes, Mcat, $K A S 3 B, F A T B 1$, and $S A D$, gradually increased.

On the other hand, the comparative analysis of the expression levels of these miRNAs and their target genes between the two cultivars showed that the expression of cpa-miR164d was significantly higher in 'M3' than in 'M8', but that that of mtr-miR156h-3p_1ss8AC was significantly lower in 'M3' than in 'M8' at the early seed developmental stage. The expression patterns of csi-miR166e-5p and Gma-MIR5368-p3_1ss21CT were similar in the developing seeds of the two cultivars of tea oil camellia. The former was similarly upregulated in the two cultivars, and its target genes, KAS1 (comp67779_c0) and S-ACP-DES6 (comp67006_c0), also showed a trend of upregulation. The latter showed low expression at the early seed developmental stage of the two cultivars, and its target gene, GAPN 
(comp67185_c0), also appeared to have a similar expression pattern in these two cultivars, with downregulated levels at the early seed developmental stage, and upregulated levels at the late seed developmental stage.

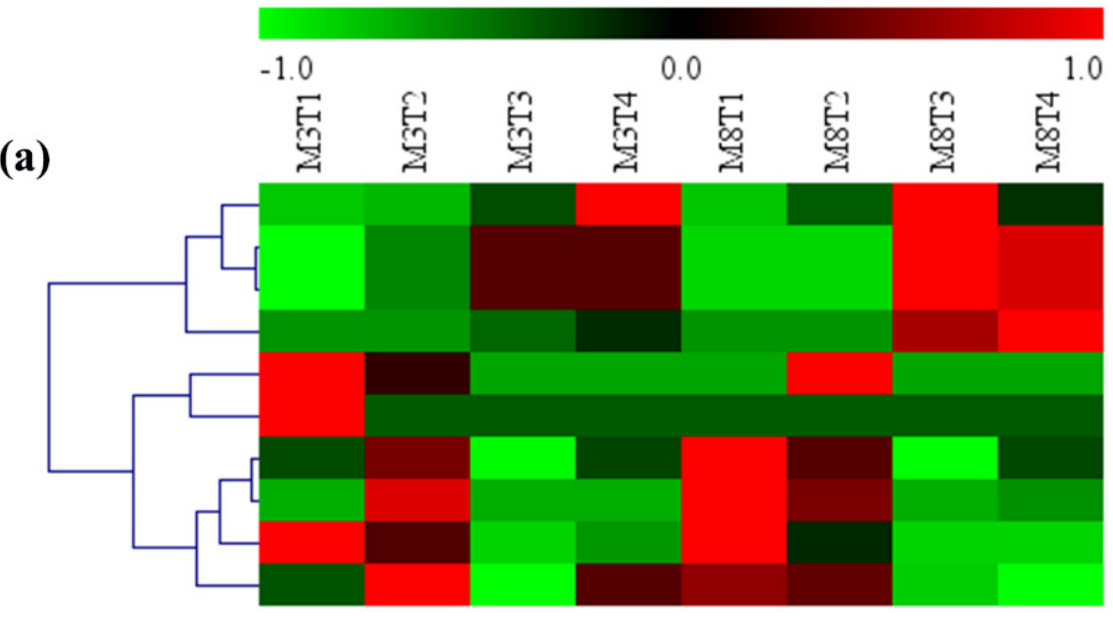

mtr-miR156h-3p_1ss8AC

csi-miR166e-5p

csi-miR166e-5p

gma-MIR5368-p3_1ss21CT

aly-miR393a-3p_1ss12TC

cpa-miR164d

tcc-miR162

rgl-miR5139 L-1

ath-miR172a

col-miRn-5p-21064_221

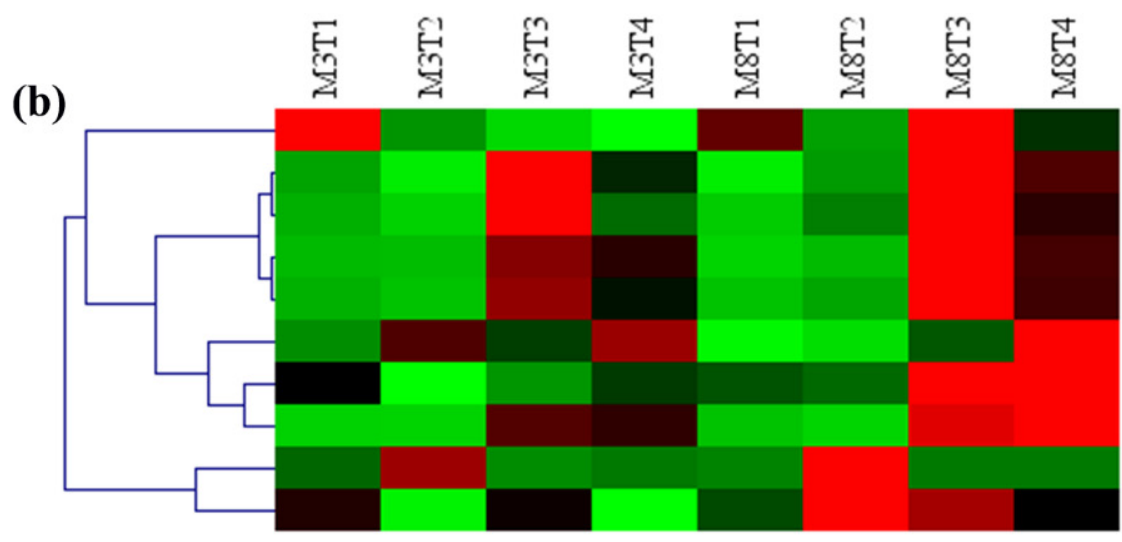

comp59939_c1 ACC1
comp61049_c0 KAS3B
comp48800_c0 $\Delta 9 D$
comp67779_c0 KAS1
comp63026_c1 Mcat
comp67006_c0 S-ACP-DES6
comp52017_c0 MOD1
comp67185_c0 GAPN
comp63911_c0 fabG
comp67050_c0 FATB1

Figure 8. Heat map analysis of (a) predicted differentially expressed miRNAs, and (b) their target genes, involved in lipid metabolism in the seeds of the 'M3' and 'M8' cultivars at four developmental stages. Red and green colors indicate high levels and low levels, respectively, of the miRNAs and their targets.

On the basis of these results, it could be speculated that these miRNAs and their target genes might be involved in the lipid metabolism pathways. The analysis of their interactions and influence in different relevant lipid metabolism pathways in tea oil camellia will provide important information for the breeding of tea oil camellia in the future.

\subsection{MiRNA-mRNA Regulation Modules Involved in Seed Size}

Seed size is one of the main factors affecting crop yield. A total of 23 miRNA-mRNA regulatory modules involved in seed size were predicted (Table 2 and Figure 9). Their target genes were transcription factors belonging to the MYB, CNR, MED, and ARF families, which play important roles in regulating seed development (Table S15). On the one hand, Mdm-miR167h_1ss22AT showed a similar expression pattern in the 'M3' and 'M8' cultivars, with low expression levels in the early seed developmental stages, and high levels in the late seed developmental stages. Its target gene, MED27 (comp135267_c0), showed the highest expression in the early seed developmental stage, and the lowest in the late seed developmental stage in the 'M3' cultivar; however, the expression of MED27 remained high at all the seed developmental stages in the 'M8' cultivar. The expression of mtr-miR156h- 
3p_1ss8AC was the lowest at the early seed developmental stage in the 'M3' and 'M8' cultivars, and its target gene, MYB44 (comp55270_c0), showed the highest expression in this stage.

Table 2. Predicted miRNAs and their target genes involved in seed size.

\begin{tabular}{|c|c|c|c|}
\hline miRNA ID & Gene ID & Gene Name & Gene Annotation \\
\hline vvi-miR160c_L+1R-1 & comp58420_c0 & ARF22 & auxin response factor 22 \\
\hline htu-miR160a_R+1 & comp58420_c0 & $A R F 22$ & auxin response factor 22 \\
\hline hpe-miR162a_L-2 & comp57222_c0 & $A R F 19$ & auxin response factor 19 \\
\hline tcc-miR162 & comp57222_c0 & ARF19 & auxin response factor 19 \\
\hline mdm-miR167h_1ss22AT & comp60627_c0 & ARF6 & auxin response factor 6 \\
\hline dpr-miR167a_R+1 & comp60627_c0 & ARF6 & auxin response factor 6 \\
\hline col-miRn-3p-16403_272 & comp57254_c0 & ARF12 & auxin response factor 12 \\
\hline mdm-miR167h_1ss22AT & comp57254_c0 & ARF12 & auxin response factor 12 \\
\hline aly-miR170-5p_1ss8CA & comp66127_c0 & ARF11 & auxin response factor 11 \\
\hline col-miRn-3p-145806_25 & comp67524_c1 & $A R F 1$ & auxin response factor 1 \\
\hline mtr-miR156h-3p_1ss8AC & comp55270_c0 & MYB44 & Transcription factor MYB44 \\
\hline ptc-miR172b-5p & comp61335_c0 & MYB44 & Transcription factor MYB44 \\
\hline ath-miR858b & comp31235_c0 & MYB44 & Transcription factor MYB44 \\
\hline col-miRn-5p-21064_221 & comp63079_c0 & MYB44 & Transcription factor MYB44 \\
\hline crt-miR168_L-2 & comp59139_c0 & MYB44 & Transcription factor MYB44 \\
\hline csi-miR166e-5p_L-1R+1_1ss21GA & comp278197_c0 & MYB82 & Transcription factor MYB82 \\
\hline ath-miR858b & comp278197_c0 & MYB82 & Transcription factor MYB82 \\
\hline ath-miR858b & comp65157_c0 & $M Y B 3$ & Transcription factor MYB3 \\
\hline mdm-miR167h_1ss22AT & comp135267_c0 & $M E D 27$ & $\begin{array}{l}\text { Mediator of RNA polymerase } \\
\text { II transcription subunit } 27\end{array}$ \\
\hline gra-miR167a_L-2R+2 & comp54724_c0 & $M E D 28$ & $\begin{array}{l}\text { Mediator of RNA polymerase } \\
\text { II transcription subunit } 28\end{array}$ \\
\hline mdm-MIR167c-p3_1ss6AG & comp63676_c0 & MED14 & $\begin{array}{l}\text { Mediator of RNA polymerase } \\
\text { II transcription subunit } 14\end{array}$ \\
\hline mes-MIR482-p3_2ss9TG21AG & comp50462_c0 & MED18 & $\begin{array}{l}\text { Mediator of RNA polymerase } \\
\text { II transcription subunit } 18\end{array}$ \\
\hline col-miRn-5p-315953_8 & comp59426_c0 & CNR2 & Cell number regulator 2 \\
\hline
\end{tabular}

On the other hand, the expression patterns of most of the miRNAs were slightly different between the two cultivars, 'M3' and 'M8', such as col-miRn-5p-315953_8, athmiR858b, and hpe-miR162a_L-2. For col-miRn-5p-315953_8, its expression was relatively low in four seed development stages of the 'M8' cultivar but was low only in the first to third developmental stages of the 'M3' cultivar, being high in the late seed developmental stage. Its target gene, CNR2 (comp59426_c0), showed low expression in the late seed developmental stage, but the highest expression in the third seed developmental stage in the 'M3' cultivar; however, the expression of CNR2 was the lowest in the third seed developmental stage in the 'M8' cultivar.

For ath-miR858b, its expression was lower in the developing seeds in the 'M8' cultivar than in the 'M3' cultivar. The expression of ath-miR858b was the highest in the early seed developmental stage in the 'M3' cultivar, and then gradually decreased; however, its lowest expression in the 'M8' cultivar appeared in the third developmental stage, and then gradually increased. The expression of its two target genes, MYB82 (comp278917_c0) and MYB3 (comp65157_c0), showed a general decline in the developing seeds of the 'M3' and 'M8' cultivars; however, MYB3 showed the lowest expression in the third seed developmental stage, and then slightly increased. In the third seed developmental stage, the expression of MYB3 was lower in 'M3' than in 'M8', and its rate of increase in 'M3' was slower than that in 'M8' from the third to the fourth seed developmental stages. In addition, the expression of MYB44 (comp31235_c0), another target of ath-miR858b, showed a slight difference between the 'M3' and 'M8' cultivars. In the 'M8' cultivar, MYB44 consistently maintained high expression in all the seed development stages, but in the 'M3' cultivar, its 
expression was the highest in the early seed developmental stage, and the lowest in the second seed developmental stage, before it gradually increased.

(a)

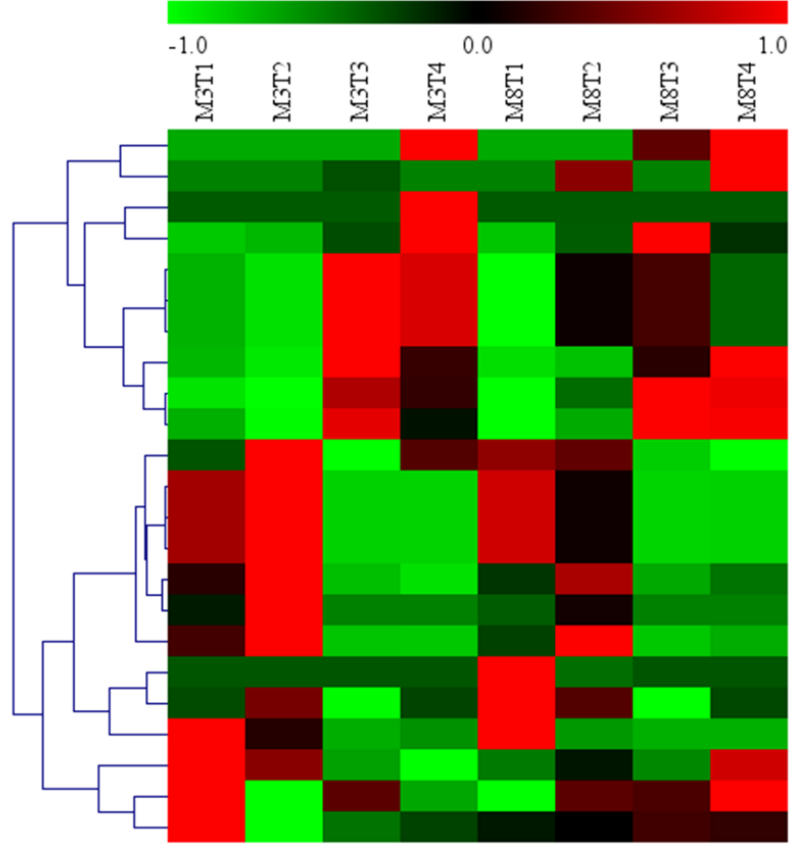

col-miRn-3p-145806 25

crt-miR168 L-2

col-miRn-5p-315953_8

mtr-miR156h-3p_1ss8AC

mdm-miR167h_1ss22AT

mdm-miR167h_1ss22AT

mdm-miR167h 1ss22AT

aly-miR170-5p_1ss8CA

gra-miR167a L-2R+2

dpr-miR167a $\mathrm{R}+1$

col-miRn-5p-21064_221

ath-miR858b

ath-miR858b

ath-miR858b

mdm-MIR167c-p3 1ss6AG

col-miRn-3p-16403 272

csi-miR166e-5p_L-1R+1_1ss21GA

hpe-miR162a L-2

tcc-miR162

ptc-miR $172 b-5 \mathrm{p}$

mes-MIR482-p3 2ss9TG21AG

htu-miR160a_R+1

vvi-miR160c_L+1R-1

(b)

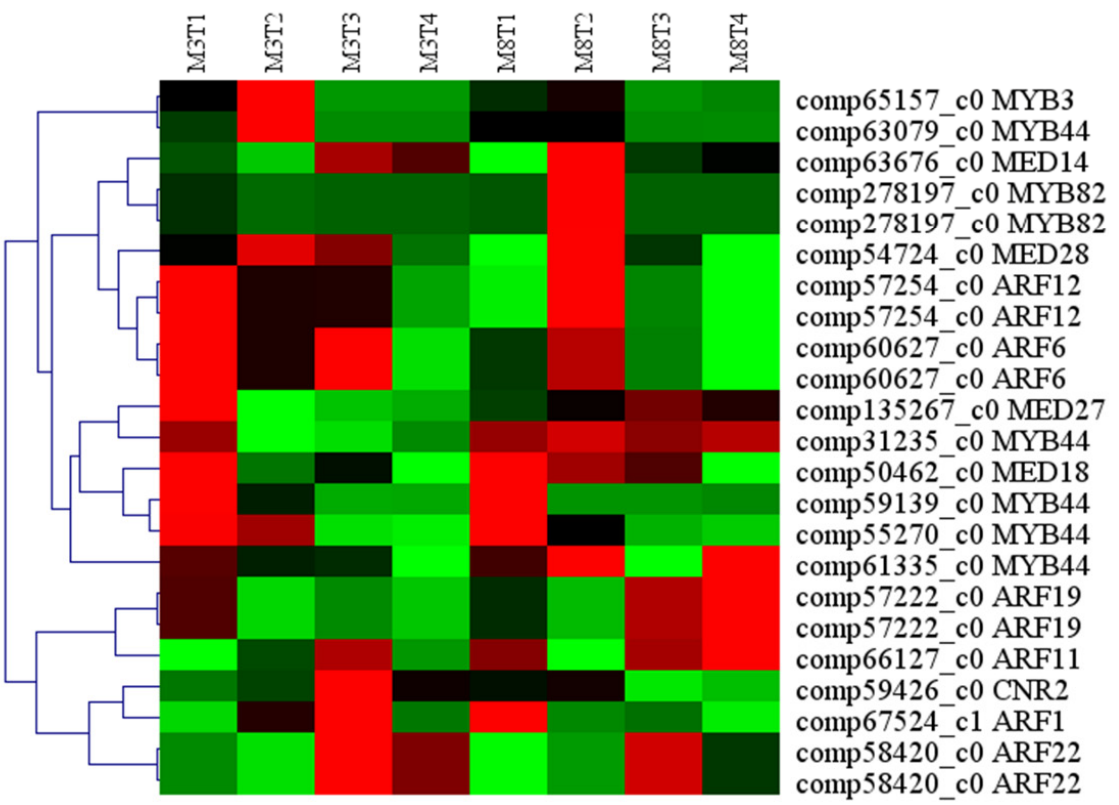

Figure 9. Heat map analysis of (a) predicted differentially expressed miRNAs, and (b) their target genes, involved in seed development from seeds of the 'M3' and 'M8' cultivars at four developmental stages. Red and green colors indicate high levels and low levels, respectively, of the miRNAs and their targets.

The expression of hpe-miR162a_L-2 was low in the developing seeds of the 'M3' cultivar, but high in the early seed developmental stage, and then it gradually decreased in the 'M8' cultivar. Its target gene, ARF19 (comp57222_c0), had a similar expression pattern in the developing seeds of the 'M3' and 'M8' cultivars; its expression was high in the early seed developmental stage, but gradually decreased in the second seed developmental stage, 
and then its expression increased in the third seed developmental stage, and maintained the same levels from the third to fourth seed developmental stages.

\subsection{MiRNA-mRNA Regulatory Module Involved in Growth and Development}

According to the target prediction annotation information, 12 key miRNAs and their target genes related to growth and development (Table S15) were identified in the highand low-oil cultivars of tea oil camellia. On the basis of the heat map analysis, the genes targeted by relevant miRNAs were ubiquitously expressed in the developing seeds of tea oil camellia, and the different miRNA expression levels were significantly different between the 'M3' and 'M8' cultivars (Table 3 and Figure 10). On the one hand, the expression pattern of han-miR156a_L+1 was similar in the 'M3' and 'M8' cultivars, with significantly low expression levels in the early seed developmental stage, that sharply increased to the highest levels in the late seed developmental stage. Its two target genes, SPL4 (comp60372_c0) and SBP2 (comp62492_c0), had the highest expression in the early seed developmental stage in the two cultivars, and the lowest in the late developmental stage. The expression pattern of rco-miR156e_L+1R-1 was similar to that of han-miR156a_L+1, but the expression patterns of its three target genes, ACS1 (comp66521_c0), SBP2 (comp62492_c0), and SBP1 (comp61590_c1), showed a downward trend in the developing seeds of the two cultivars.

Table 3. Predicted miRNAs and their target genes involved in growth and development.

\begin{tabular}{cccc}
\hline miRNA ID & Gene ID & Gene Name & Gene Annotation \\
\hline ccl-miR171 & comp61081_c0 & SCL16 & Scarecrow-like protein 6 \\
ssl-miR171b_2ss11CT19AT & comp61081_c0 & SCL16 & Squarecrow-like protein 6 \\
han-miR156a_L+1 & comp60372_c0 & SPL4 & Squamosa promoter-binding protein 2 \\
han-miR156a_L+1 & comp62492_c0 & SBP2 & 1-aminocyclopropane-1-carboxylate synthase \\
rco-miR156e_L+1R-1 & comp66521_c0 & Squamosa promoter-binding protein 2 & Squamosa promoter-binding protein 1 \\
rco-miR156e_L+1R-1 & comp62492_c0 & Transcription factor TCP24 \\
rco-miR156e_L+1R-1 & comp61590_c1 & SBP1 & transport inhibitor response 1 \\
mtr-miR156h-3p_1ss8AC & comp65957_c0 & TCP24 & Protein AUXIN SIGNALING F-BOX 2 \\
cpa-miR393_R-1 & comp61948_c0 & AFB2 & Zinc finger protein CONSTANS-LIKE 13 \\
cpa-miR393_R-1 & comp64823_c0 & EOLEBP-like factor & ERF115
\end{tabular}

On the other hand, the expression of some, such as mtr-miR156h-3p_1ss8AC and col-miRn-3p-9080_426, showed significant differences between the 'M3' and 'M8' cultivars. For col-miRn-3p-9080_426, its expression was gradually upregulated in the 'M8' cultivar from the early to third seed developmental stages but was the lowest in the fourth seed developmental stage. By contrast, col-miRn-3p-9080_426 consistently showed higher expression in all the seed developmental stages in the 'M3' cultivar than in the 'M8' cultivar. Its one target gene, COL13 (comp63752_c0), was downregulated in the two cultivars in the late seed developmental stage. The expression pattern of another target gene, ERF115 (comp58165_c0), was similar to that of the COL13 gene in the 'M3' cultivar, but in the 'M8' cultivar, the expression of ERF115 was the highest in the early seed developmental stage and decreased rapidly to a low level in the third seed developmental stage; after this, it showed a slight increase in the late seed developmental stage. For mtr-miR156h-3p_1ss8AC, its expression was significantly lower in the 'M3' cultivar than in the 'M8' cultivar in the early developmental stage, and it increased slowly after the early seed developmental stage in the 'M3' cultivar; however, it was consistently high throughout all the seed developmental stages in the 'M8' cultivar. TCP24 (comp65957_c0), a target gene of miR156h-3p_1ss8AC, had high expression in the 'M3' cultivar in the early seed developmental stage, but it was consistently low in the 'M8' cultivar in all the seed developmental stages. 
(a)

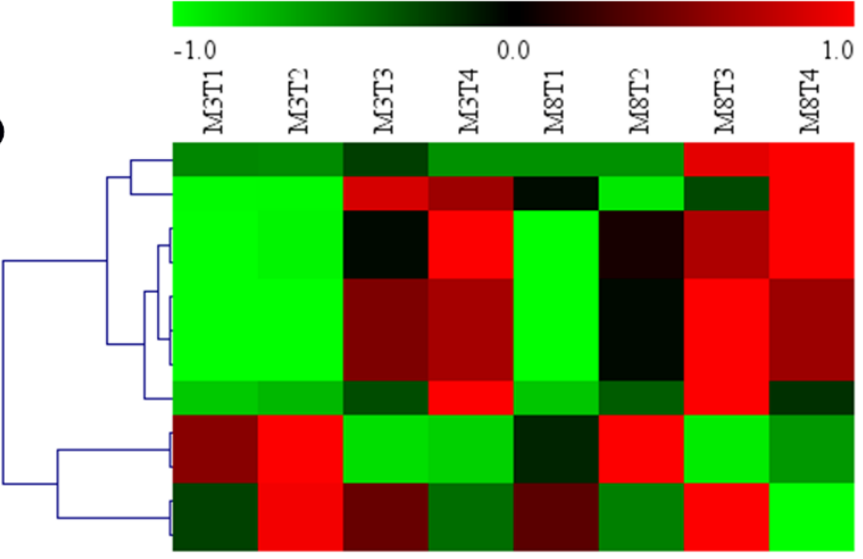

ccl-miR171

ssl-miR171b_2ss11CT19AT

han-miR156a $\mathrm{L}+1$

han-miR156a L+1

rco-miR $156 \mathrm{e}$ L+1R-1

rco-miR $156 \mathrm{e} \mathrm{L}+1 \mathrm{R}-1$

rco-miR $156 \mathrm{e}^{-} \mathrm{L}+1 \mathrm{R}-1$

mtr-miR156h-3p_1ss8AC

cpa-miR393 R-1

cpa-miR393_R-1

col-miRn-3p-9080_426

col-miRn-3p-9080_426

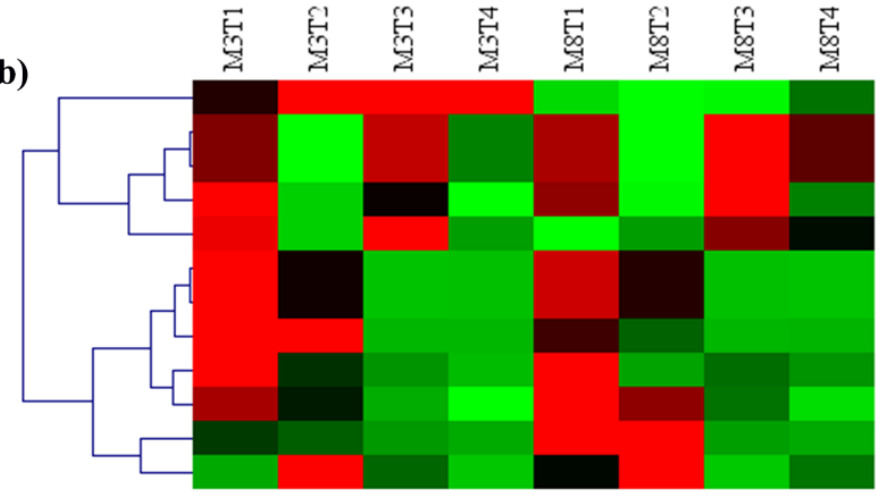

comp65957 c0 TCP24

comp61081_c0 SCL16

comp61081 c0 SCL16

comp61948_c0 T1R1

comp64823 ${ }^{-} 0$ AFB2

comp62492_c0 SBP2

comp62492 c0 SBP2

comp61590_c1 SBP1

comp 60372 c0 SPL4

comp63752_c0 COL13

comp66521 c0 ACS1

comp58165_c0 ERF115

Figure 10. Heat map analysis of (a) predicted differentially expressed miRNAs, and (b) their target genes, involved in the growth and development of seeds from the 'M3' and 'M8' cultivars at four developmental stages. Red and green colors indicate high levels and low levels, respectively, of the miRNAs and their targets.

According to these results, these identified miRNAs and their target genes show highly correlative relationships between their expression levels during seed development, and the differences in the expression levels between the two cultivars, or among the different seed developmental stages, might exert a great influence on the growth and development of tea oil camellia seeds.

\subsection{MiRNA-mRNA Regulatory Modules Involved in Resistance, Yield, and Quality}

According to the enrichment analysis of the target genes of the differentially expressed miRNAs, 11 miRNA-mRNA regulatory modules related to stress resistance were identified, mainly involving two categories: abiotic stresses and biotic stresses (Table 4 and Table S15). In addition, two miRNA-mRNA regulatory modules, which may affect the yield and quality of tea oil camellia seed, were also predicted (Table 4 and Table S15). Their expression patterns and levels appeared to be obviously different between the 'M3' and 'M8' cultivars, and among different seed developmental stages (Figure 11). On the one hand, the expression of han-miR156a_L 1 in the 'M3' and 'M8' cultivars was the lowest in the early seed developmental stage, and then increased gradually; accordingly, its target gene, SPL16 (comp56544_c0), showed the highest expression in the early seed developmental stage, and then decreased in these two cultivars. 
Table 4. Predicted miRNAs and their target genes involved in resistance, yield, and quality.

\begin{tabular}{|c|c|c|c|}
\hline miRNA ID & Gene ID & Gene Name & Gene Annotation \\
\hline col-miRn-3p-16403_272 & comp14497_c0 & BAM1 & $\begin{array}{l}\text { leucine-rich repeat receptor-like } \\
\text { serine/threonine-protein kinase, BAM1 }\end{array}$ \\
\hline col-miRn-3p-16403_272 & comp47735_c0 & Os03g0214200 & Ninja-family protein, Os03g0214200 \\
\hline col-miRn-5p-28629_169 & comp66741_c0 & ERF106 & EREBP-like factor \\
\hline ath-miR858b & comp31687_c0 & MYB4 & Myb-related protein, Myb4 \\
\hline ath-MIR5645b-p5_2ss19TG21TC & comp59571_c0 & PYL1 & Abscisic acid receptor, PYL1 \\
\hline mtr-miR156h-3p_1ss8AC & comp63682_c0 & $B A G 7$ & BAG family molecular chaperone regulator 7 \\
\hline mtr-miR156h-3p_1ss8AC & comp66713_c0 & NRT1.7 & $\begin{array}{l}\text { proton-dependent oligopeptide transporter, } \\
\text { POT family }\end{array}$ \\
\hline mdm-MIR167c-p3_1ss6AG & comp56349_c0 & $\mathrm{XDH} 1$ & xanthine dehydrogenase/oxidas \\
\hline gra-miR167a_L-2R+2 & comp62927_c0 & APUM5 & Pumilio homolog 5 \\
\hline gma-miR394a-5p_R+1_1ss18TA & comp64503_c0 & SGS3 & Protein SUPPRESSOR OF GENE SILENCING 3 \\
\hline nta-MIR6149a-p5_2ss18CT21TA & comp65951_c0 & CPR30 & F-box protein CPR30 \\
\hline han-miR156a_L+1 & comp56544_c0 & SPL16 & Squamosa promoter-binding-like protein 16 \\
\hline ppe-miR172a-5p_1ss21GA & comp65585_c0 & PAB2 & $20 \mathrm{~S}$ proteasome subunit alpha 2 \\
\hline
\end{tabular}

(a)

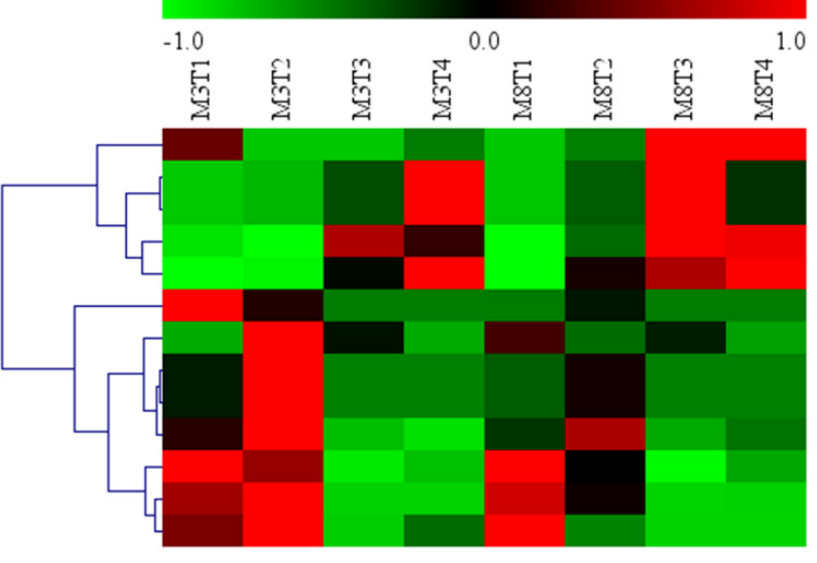

ath-MIR 5645b-p5 2ss19TG21TC

mtr-miR156h-3p_1ss8AC

mtr-miR156h-3p_1ss8AC

gra-miR167a L-2R+2

han-miR156a L+1

gma-miR394a-5p $R+1$ 1ss18TA

col-miRn-5p-28629_169

col-miRn-3p-16403-272

col-miRn-3p-16403 272

mdm-MIR167c-p3_ 1ss6AG

nta-MIR6149a-p5 2ss18CT21TA

ath-miR858b

ppe-miR172a-5p_1ss21GA

(b)

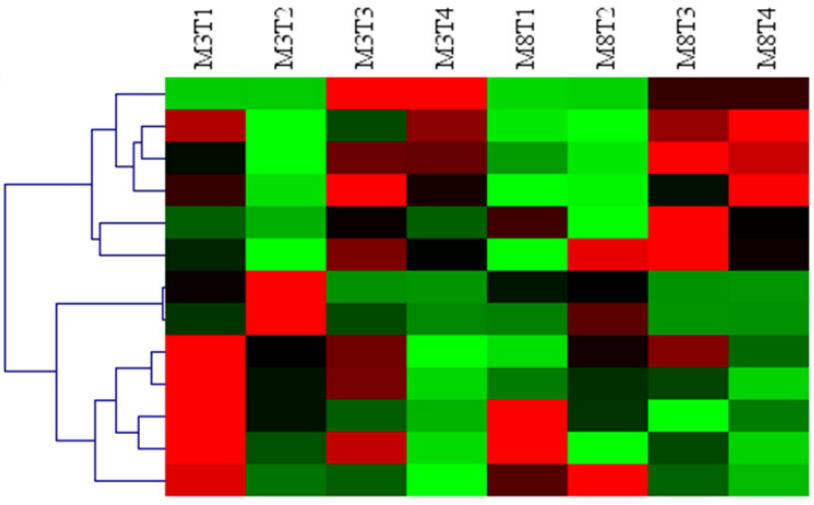

comp59571_c0 PYL1

comp47735 c0 Os03g0214200

comp62927_c0 APUM5

comp 64503 c0 SGS3

comp14497 c0 BAM1

comp65951_c0 CPR30

comp66741 c0 ERF106

comp31687_c0 MYB4

comp66713 c0 NRT1.7

comp56349_c0 XDH1

comp63682 c0 BAG7

comp56544 c0 SPL16

comp65585_c0 PAB2

Figure 11. Heat map analysis of (a) predicted differentially expressed miRNAs, and (b) their target genes, related to resistance, yield, and quality from the seeds of the 'M3' and 'M8' cultivars at four developmental stages. Red and green colors indicate high levels and low levels, respectively, of the miRNAs and their targets.

On the other hand, the expression patterns of ath-miR858b and nta-MIR6149a-p5 2ss18CT21TA were different in the high- and low-oil cultivars. Their expression was consistently high in the 'M3' cultivar throughout the seed developmental stages, but for the 'M8' cultivar, the expression was high in the early and second seed developmental stages, 
and then gradually decreased to the lowest value in the third seed developmental stage, and then slowly increased. Accordingly, the expression of MYB4 (comp31687_c0), a target gene of ath-miR858b, was the lowest in the 'M8' cultivar in the fourth seed developmental stage. The expression of CPR30 (comp65951_c0), a target gene of nta-MIR6149a-p5_2ss18CT21TA, was the lowest in the 'M3' cultivar in the second seed developmental stage, but its highest expression in the 'M8' cultivar appeared in the third seed developmental stage.

For ppe-miR172a-5p_1ss21GA, its expression remained high in the 'M3' cultivar in all the seed developmental stages, but in the 'M8' cultivar, its expression was only high in the early seed developmental stage, and then significantly decreased and reached its lowest expression level in the fourth seed developmental stage. Its target gene, $P A B 2$, showed the lowest expression in the 'M3' cultivar in the late seed developmental stage, and its highest expression appeared in the early seed developmental stage in the 'M8' cultivar.

For mtr-miR156h-3p_1ss8AC, its expression remained consistently high in the 'M8' cultivar throughout the four different seed developmental stages, but in the ' $\mathrm{M} 3$ ' cultivar, its expression was the lowest in the early seed developmental stage, and then gradually increased. However, its two target genes, BAG7 (comp63682_c0) and NRT1.7 (comp66713_c0), showed low expression in the 'M3' cultivar in the early seed developmental stage, which then gradually decreased to the lowest level in the late seed developmental stage. Although the expression of these two target genes was also the lowest in the 'M8' cultivar in the late seed developmental stage, it was still higher than that in the 'M3' cultivar.

\subsection{Validation of Expression Levels of miRNAs and Its Target mRNAs by qRT-PCR}

To validate the high-throughput sequencing data, six regulatory modules of differentially expressed miRNAs and their target genes were selected, including han-miR156a_L+1-SPL4, rco-miR156e_L+1R-1-SBP1, mtr-miR156h-3p_1ss8AC-TCP24, ppe-miR172a-5p_1ss21GA-PAB2, nta-MIR6149a-p5_2ss18CT21TA-CPR30, and col-miRn-3p-9080_426-ERF115. Their expression patterns and levels in the high- and low-oil cultivars of tea oil camellia at the four different seed developmental stages were identified by qRT-PCR analysis (Figure 12). The results show that six miRNA-mRNA regulatory modules had differential expression, and the expression patterns of most of the miRNAs and their target genes were consistent with the results obtained in the high-throughput small RNA sequencing data. All six selected miRNAs showed opposite expression trends relative to their target genes.

Han-miR156a_L 1 was upregulated in high- and low-oil cultivars of tea oil camellia, and its expression was the lowest in the early seed developmental stage, and highest in the late developmental stage (Figure 12a). The expression of han-miR156a_L 1 in the 'M3' cultivar was higher than that in the 'M8' cultivar; by contrast, the expression of its target gene, SPL4, was the highest in the early seed developmental stage, and then decreased sharply to the lowest level in the late seed developmental stage (Figure 12a). The expression of rco-miR156e_L 1R-1 was upregulated in the 'M8' cultivar; it gradually increased from the early to third seed developmental stages, peaking in the third seed developmental stage, and then decreasing slightly (Figure 12b). The expression of rco-miR156e_L 1R-1 in the 'M3' cultivar was upregulated continuously and reached the highest value in the late seed developmental stage, but always remained lower than in the 'M8' cultivar (Figure 12a). Interestingly, the target gene of rco-miR156e_L 1R-1, SBP1, was downregulated in the 'M3' and 'M8' cultivars, and its expression was the highest in the early seed developmental stage, and lowest in the late developmental stage (Figure 12b).

The expression of mtr-miR156h-3p_1ss8AC was upregulated in the 'M3' cultivar, and was significantly lower than that in the 'M8' cultivar in the early seed developmental stage; after this, it showed a rapidly increasing trend, with the highest expression in the late seed developmental stage (Figure 12c). By contrast, the expression of mtr-miR156h-3p_1ss8AC in the 'M8' cultivar was relatively high in all the seed developmental stages. Surprisingly, the expression of the target gene of mtr-miR156h-3p_1ss8AC, TCP24, in the 'M3' cultivar, was also low in the early seed developmental stage (Figure 12c), but was then rapidly upregulated; however, its expression in the 'M8' cultivar remained low in all the seed 
developmental stages (Figure 12c). The expression of miR156h-3p_1ss8AC was found to be negatively correlated with the expression of its target gene in the low-oil 'M8' cultivar.
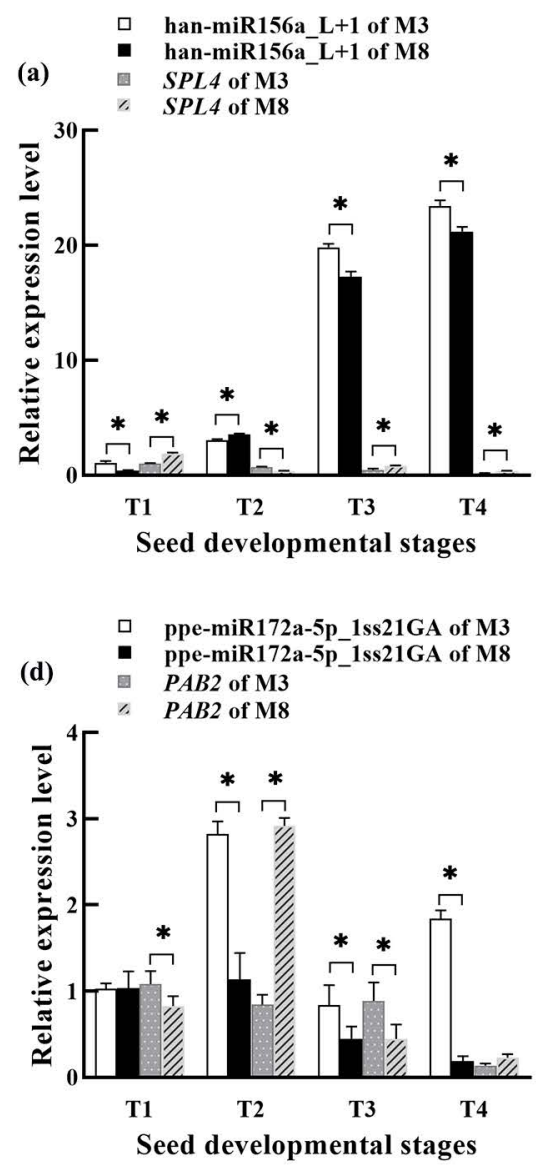
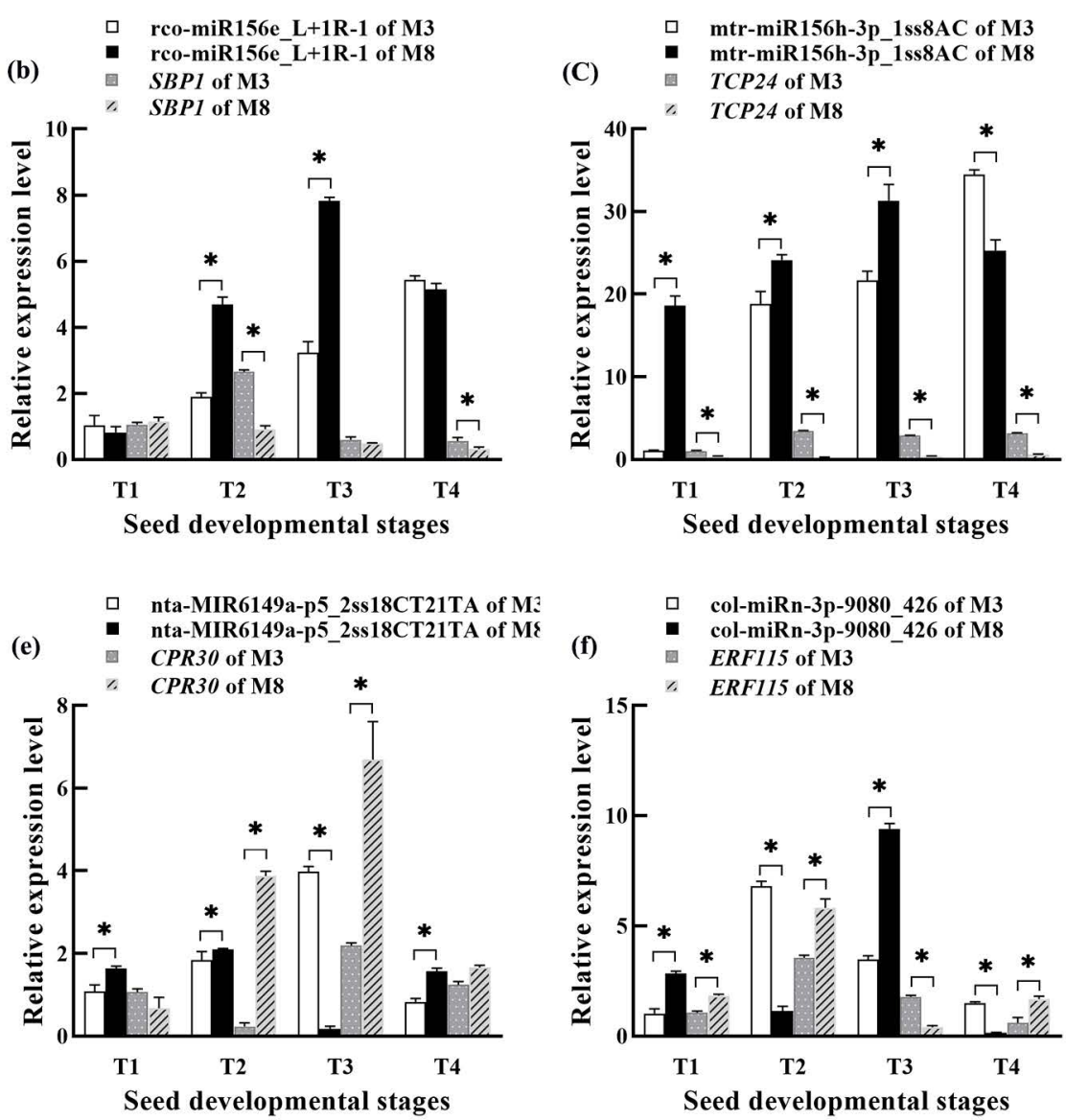

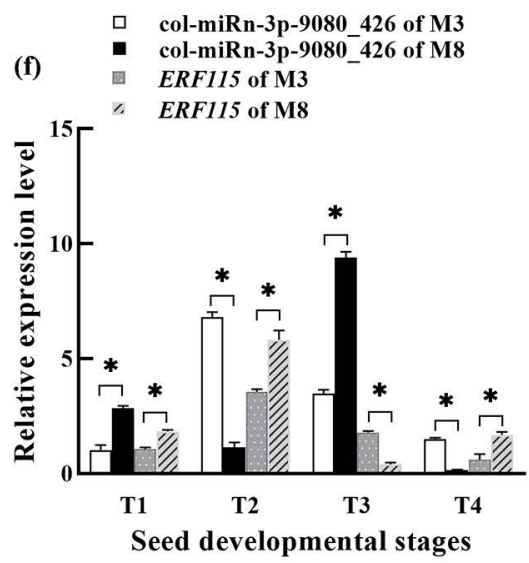

Figure 12. Expression differences of selected miRNA-mRNA pairs in the seeds of the ' $M 3$ ' and 'M8' cultivars of tea oil camellia at four different developmental stages: (a) han-miR156a_L+1-SPL4; (b) rcomiR156e_L+1R-1-SBP1; (c) mtr-miR156h-3p_1ss8AC-TCP24; (d) ppe-miR172a-5p_1ss21GA-PAB2; (e) nta-MIR6149a-p5_2ss18CT21TA-CPR30; and (f) col-miRn-3p-9080_426-ERF115. The error bars indicate standard deviations for the three biological replicates. ${ }^{*}$ represents significant differences between the two cultivars at the same seed developmental stage, on the basis of a Student's $t$-test at $p<0.05$.

The expression of ppe-miR172a-5p_1ss21GA was the highest in the 'M3' cultivar in the second seed developmental stage (Figure 12d), while the expression of its target gene, $P A B 2$, was lower in the second seed developmental stage than in the early and third seed developmental stages. The expression pattern of ppe-miR172a-5p_1ss21GA was similar to that of its target gene in the 'M8' cultivar, and both were first upregulated and then downregulated (Figure 12d). The expression of ppe-miR172a-5p_1ss21GA was found to be negatively correlated with the expression of its target gene in the high-oil ' $\mathrm{M} 3$ ' cultivar.

Nta-MIR6149a-p5_2ss18CT21TA had the highest expression in the 'M3' cultivar in the third seed developmental stage, but its expression was the lowest in the 'M8' cultivar in the third seed developmental stage and was significantly lower than that in the 'M3' cultivar (Figure 12e). Its target gene, CPR30, was low in the 'M3' cultivar in the fourth developmental stage. The expression of the CPR30 gene in the 'M3' cultivar was higher than that in the 'M8' cultivar in the early seed developmental stage but was lower in the 'M3' cultivar than in the 'M8' cultivar in the other three seed developmental stages (Figure 12e). The expression of col-miRn-3p-9080_426 peaked in the 'M3' cultivar in the second seed developmental stage, and in the 'M8' cultivar in the third seed developmental 
stage (Figure 12f). It was first upregulated and then downregulated in the 'M3' cultivar. The target gene, ERF115, of nta-MIR6149a-p5_2ss18CT21TA, showed the lowest expression in the 'M8' cultivar in the third seed developmental stage, and its expression first increased and then decreased in the low-oil 'M3' cultivar (Figure 12f).

The above results, obtained by qRT-PCR validation, are consistent with the sequencing results for the expression patterns and levels of miRNAs and their targeted genes (Figure 12), indicating the accuracy and high efficiency of the small RNA high-throughput sequencing results, and reflecting the dependability of the qRT-PCR method used to identify the temporal and spatial expression characteristics and expression differences of related functional miRNAs and target genes in tea oil camellia at different seed developmental stages.

3.10. Validation of Targeting Relationship of the cpa-miR393_R-1-AFB2 Regulatory Module by Luciferase Assays

A target prediction analysis server, psRNA target [56], was used to assess the complementarity between cpa-miR393_R-1 and the target site of AFB2, with the default parameters and a maximum expectation value of 4 (the number of mismatches allowed). The potential of cpa-miR393_R-1 to target the AFB2 3'-UTR was predicted (Figure 13a). The luciferase activity in the HEK-293T cells cotransfected with the cpa-miR393_R-1 recombinant expression vector, and the expression vector containing the $3^{\prime}$-UTR of AFB2 fused with the reporter gene was decreased by nearly $36.26 \%$ (Student's $t$-test, $p<0.05$ ) compared to that in the control group (Figure 13b). These results indicate that the AFB2 gene was targeted by cpa-miR393_R-1.

a cpa-miR393_R-1 1 TCCAAAGGGATCGCATTG-ATC 21 ||||||||||||||||$|* *| \mid$

AFB2 1579 AGGTTTCCCTAGCGTAACABAG 1558

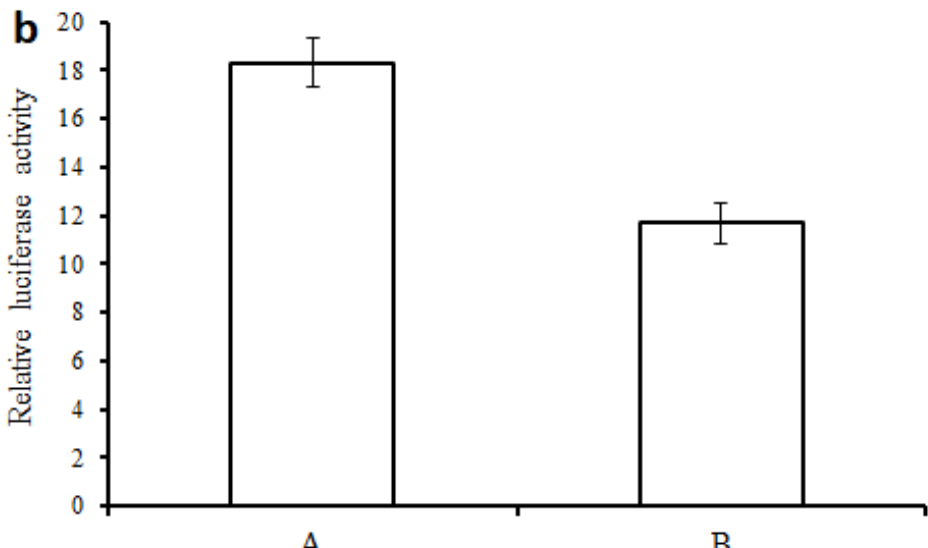

A

B

Figure 13. The validation of cpa-miR393_R-1 and target AFB2: (a) prediction of the binding sites of cpa-miR393_R-1 in AFB2 mRNA using psRNATarget; (b) effect of cpa-miR393_R-1 expression on luciferase activity in transfected cells: (A) pCDNA3.1 + pmirGLO-AFB2, and (B) pCDNA3.1cpa-miR393_R-1 + pmirGLO-AFB2. Data are represented as the means \pm standard deviations (SDs) from three independent experiments.

\section{Discussion}

\subsection{Identification of miRNAs in Developing Seeds of Tea Oil Camellia}

In this study, a total of 196 miRNAs, including 156 known and 40 novel miRNAs, were identified in the developing seeds of the 'M3' and 'M8' cultivars of tea oil camellia. For the 196 miRNAs identified in the seeds of tea oil camellia, a total of 17,166 target genes were predicted, and there were multiple target genes targeted by the same miRNA, and 
the same gene was targeted by several miRNAs. This is consistent with the report that one miRNA can regulate two or more target genes [57], and that the same target gene can also be targeted by different miRNAs [58]. The 196 miRNAs identified in tea oil camellia seeds appeared most frequently in the gma species; most of the conserved miRNAs were from 35 miRNA families, such as miR160, miR156, miR159, miR162, and miR164. This also demonstrates that miRNAs are highly conserved in their evolutionary relationships, especially in plants [59].

The exploration of the molecular mechanisms of miRNA regulation involved in various biological pathways in plants is a field of great interest to current researchers [60]. A total of 33,418 miRNA-mRNA regulatory modules were predicted in this study, 25,988 of which were related to different biological processes, including 21,132 miRNA-mRNA modules involved in different cell components, and 4675 modules involved in molecular function. In these, 107 significantly enriched GO terms were related to the nucleus, sequence-specific DNA binding, the regulation of transcription, the DNA-template, and electron carrier activity. In addition, 31 significantly enriched KEGG pathways were mainly involved in pyruvate metabolism, the regulation of the actin cytoskeleton, fatty acid biosynthesis, and the citrate cycle. These results indicate that miRNAs regulate different biological functions and metabolic pathways in the developing seeds of tea oil camellia. Excavating these important miRNA-mRNA regulatory modules could enable a clear understanding of the biological functions and metabolic pathways of miRNA-regulated target genes during seed development in this species, providing favorable resources for improving tea oil camellia in the future.

\subsection{MiRNA-mRNA Modules Involved in Lipid Metabolism in Tea Oil Camellia}

Studies have shown that miRNAs are involved in lipid metabolism by regulating targeted genes in oil crops, such as B. napus [61], hazel [36], oil palm [37], olive [38], peony [22], walnut [10], and yellowhorn [11]. In this study, the mtr-miR156h-3p_1ss8AC had high expression in the developing seeds of tea oil camellia and targeted the fabG gene. The $f a b G$ gene encodes a member of the ketoacyl reductase family of proteins, and it is an essential enzyme for type II fatty acid biosynthesis [62]. This is consistent with miR156 influencing the lipid biosynthesis of the seeds in sea buckthorn [24] and rapeseed [63].

A total of 10 miRNA-mRNA regulatory modules related to lipid metabolism pathways in the seeds of tea oil camellia were first found in this study (Figure 14). The regulation module of tcc-miR162-ACC1 may be related to the oil and seed yield of tea oil camellia. This is because ACCase is a rate-limiting enzyme that controls the flow of carbon to fatty acid synthesis; not only is it a key gene for lipid and fatty acid biosynthesis in tea oil camellia [64], but its high expression is also closely related to the high yield and oil content of oil palm $[65,66]$. The GAPN gene is related to lipid accumulation [67]; thus, the gmaMIR5368-p3_1ss21CT-GAPN regulatory module is involved in lipid metabolism in tea oil camellia.

The remaining regulation modules of csi-miR166e-5p-KAS1/S-ACP-DES6, rgl-miR5139_ L-1-KAS3B, cpa-miR164d-Mcat, col-miRn-5p-21064_221-FATB1, aly-miR393a-3p_1ss12TC $M O D 1$, and ath-miR172a- $\triangle 9 D$ were related to the fatty acid biosynthesis metabolic pathway, because these target genes, such as KASIII [68], Mcat [69], MOD1 [70], and $\triangle 9 D$ [24], reported in previous studies (Table S15), are related to lipid biosynthesis and fatty acid formation and accumulation. KASI is involved in the formation of C16 or C18 saturated fatty acids catalyzed by the fatty acid synthase complex [71]. S-ACP-DES6 (comp67006_c0) regulates the contents of oleic acid in the seeds of tea oil camellia. The expression of csimiR166e-5p was similarly upregulated in the 'M3' and 'M8' cultivars, and its target gene, $S-A C P-D E S 6$, had a trend of upregulation that promoted the formation and accumulation of oleic acid in developing seeds. This is consistent with the result from a previous report on the $S-A C P$-DES gene, stating that it is a key gene involved in oleic acid biosynthesis in tea oil camellia [72] and Arabidopsis [73]. Previous studies found that the overexpression of the FATB1 gene in Arabidopsis seeds resulted in a four-fold increase in the palmitic acid content 
in the seed oil [74]. This indicates that the regulation module of col-miRn-5p-21064_221FATB1 in tea oil camellia may be related to fatty acid formation and accumulation in its seed oil.

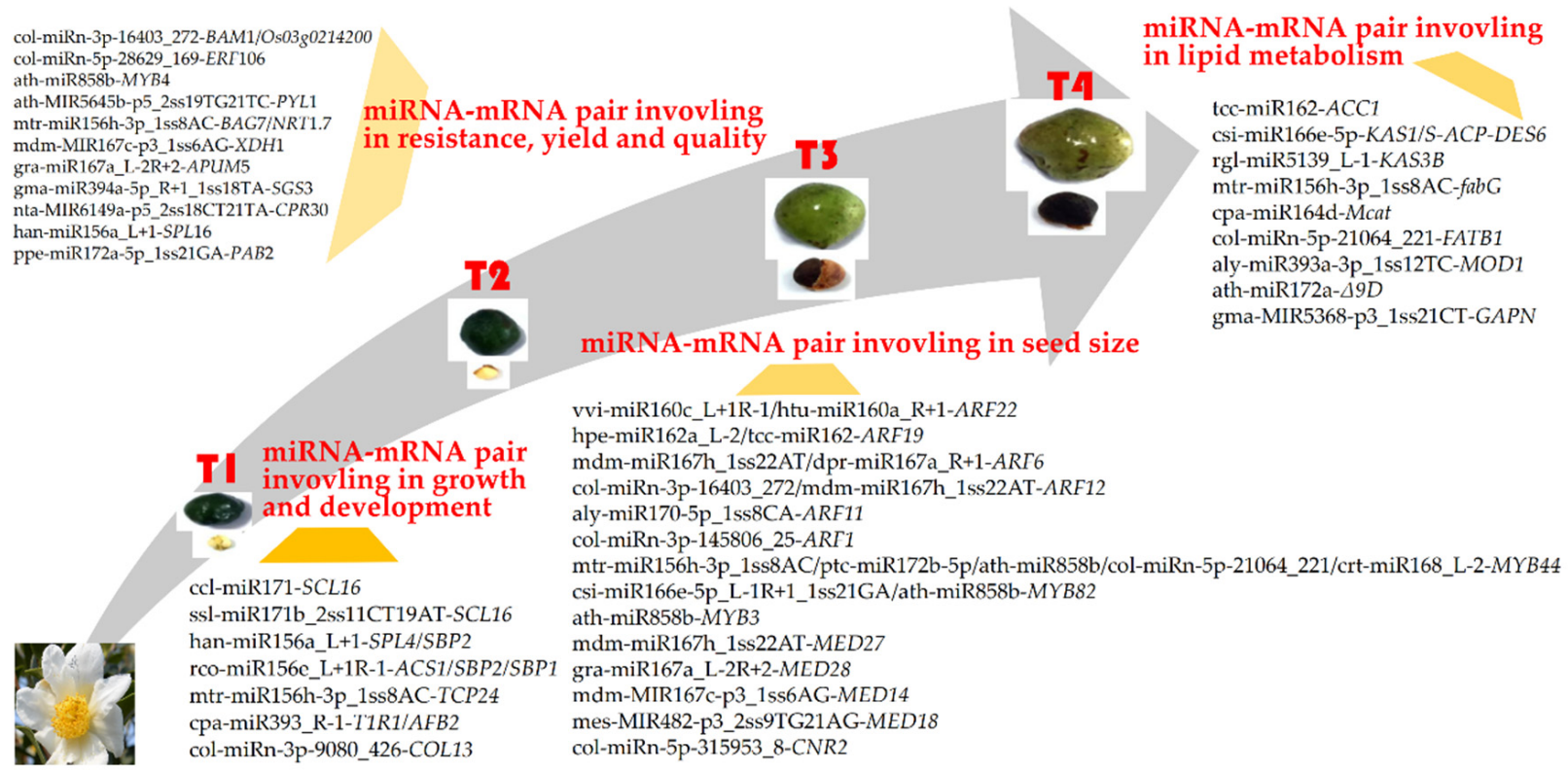

Figure 14. miRNA-mRNA regulatory modules in developing seeds of tea oil camellia.

The analysis of the expression patterns and expression levels for the miRNA-mRNA regulatory modules involved in lipid metabolism provides a clear perspective for understanding the role of miRNAs in the lipid biosynthesis of tea oil camellia seeds, indicating the potential for the utilization of miRNAs to improve the seed oil content and oil components in tea oil camellia in the future.

\subsection{MiRNA-mRNA Regulatory Modules Involved in Seed Size in Tea Oil Camellia}

In this study, our data show that some target genes in the 23 miRNA-mRNA regulatory modules in the seeds of tea oil camellia were transcription factors (e.g., ARF, CNR, MYB, and MED), which are related to the seed size (Table S15). This is consistent with most of the target genes encoding transcription factors in miRNA-mRNA modules related to seed size [18,75], mainly including ARF [24,29], CNR [76], and MYB [77]. In chickpea and oilseed crops, transcription factors of the MYB and ARF families are involved in seed size/weight determination $[29,30]$. Negative regulatory modules of hpe-miR162a_L-2-ARF19 may be involved in the seed development of tea oil camellia because the low expression of hpemiR162a_L-2 and the high expression of ARF19 in the early seed development stage are helpful for auxin biosynthesis. The function of SIMYB is as a candidate gene involved in the fruit set process in tomatoes [78]. In tea oil camellia, ath-miR858b may regulate seed development by targeting MYB transcription factors. In the developing seeds of tea oil camellia, the three transcription factors, MYB82 (comp278917_c0), MYB3 (comp65157_c0), and MYB44 (comp31235_c0), cotargeted by ath-miR858b, showed a declining trend and lower expression in the two cultivars of 'M3' and 'M8', and the seed size gradually increased in the developing seeds. This is consistent with the evidence that the transcription factor, MYB89, represses the seed yield in Arabidopsis [79].

\subsection{MiRNA-Target Genes Involved in Growth, Development, and Resistance in Tea Oil Camellia}

In this study, 12 miRNA-mRNA regulatory modules related to the growth and development of tea oil camellia seed were identified because these targeted the gene transcription factors, ACS1, AFB2, COL13, ERF115, SBP1, SBP2, SCL16, SPL4, T1R1, and TCP24, which are related to growth and development (Table S15). For example, the TCP24 modulates 
secondary cell-wall thickening and anther endothecium development [80]; the Lc-ACS1 gene might be the key gene involved in fruitlet abscission in litchis [81]; SBP1 is an important component of the atypical SCF complex, playing a unique role in the self-compatibility system of gametes [82]. An SBP2-GFP fusion protein under the control of the GmSBP2 promoter accumulates in the vascular tissues of vegetative organs, which is consistent with the proposed involvement of SBP in sucrose-transport-dependent physiological processes [83].

Han-miR156a_L+1 showed significantly low expression in the early seed developmental stage in the 'M3' and 'M8' cultivars of tea oil camellia, and then increased sharply to its highest level in the late seed developmental stage. By contrast, its two target genes, SPL4 and $S B P 2$, had the highest expression in the early seed developmental stage in two cultivars, and the lowest expression appeared in the late developmental stage. This indicated that the negative regulation modules of han-miR156a_L+1-SPL4/SBP2 promote seed development in the early seed developmental stage in tea oil camellia. This is consistent with miR156 participating in the early morphogenesis of Arabidopsis embryos by regulating the targets SPL10 and SPL11 [33], and it may also mediate the targeted regulation of SBP expression levels, affecting the flowering time in plants [29].

In tea oil camellia seeds, ccl-miR171 and ssl-miR171b_2ss11CT19AT may regulate seed development by targeting the SCL16 gene. miRNA171 controls the growth and development of flowers, roots, and stems by targeting SCL transcription factor family members in Arabidopsis [84]. The genes of AFB2 and T1R1 are auxin-receptor genes regulated by miR393, which is involved in hormone regulation and signal transduction in plants [48]. Thus, two regulation modules of cpa-miR393_R-1-AFB2/T1R1 in tea oil camellia may be related to seed development.

In addition, 13 miRNA-mRNA regulatory modules were related to resistance and yield in the seeds of tea oil camellia (Table S15), in which the target genes of APUM5 [85], BAG7 [86], CPR30 [87], ERF106 [88], MYB4 [89], Os03g0214200 [90], SGS3 [91], and SPL16 were [92] involved in resistance, and NRT1.7 was related to yield [93].

\section{Conclusions}

In conclusion, a total of 196 miRNAs, including 156 known miRNAs from 35 families, and 40 novel miRNAs, were identified in the developing seeds of high- and low-oil two cultivars of tea oil camellia, in which 55 significantly differentially expressed miRNAs were found, with 34 upregulated miRNAs, and 21 downregulated miRNAs. A total of 9 miRNAmRNA regulatory modules were related to lipid metabolism. For example, the negative regulatory module of ath-miR858b-MYB82/MYB3/MYB44 represses seed oil biosynthesis, and a regulatory module of csi-miR166e-5p-S-ACP-DES6 (comp67006_c0) was related to the formation and accumulation of oleic acid. A total of 23 miRNA-mRNA regulatory modules were involved in the regulation of seed development, such as the negative regulatory modules of hpe-miR162a_L-2-ARF19, involved in early seed development. Twelve miRNAmRNA regulatory modules regulating growth and development were identified, such as the negative regulatory modules of han-miR156a_L+1-SPL4/SBP2, which promote early seed development.

Supplementary Materials: The following supporting information can be downloaded at: https: / / www.mdpi.com/article/10.3390/cells11010071/s1, Table S1: Specific species that were related species of tea oil camellia; Table S2: Primers used for qRT-PCR of selected mature miRNAs; Table S3: Primers used for qRT-PCR of selected target genes of miRNAs; Table S4: Summary of the sequencing data from raw data to cleaned sequences from the seeds of the 'M3' cultivar of tea oil camellia; Table S5: Summary of the sequencing data from raw data to cleaned sequences from the seeds of the 'M8' cultivar of tea oil camellia; Table S6: Classifications and pre-precursors of known and novel miRNAs in developing seeds of tea oil camellia in this study; Table S7: Length distribution of unique miRNAs; Table S8: Known miRNAs in tea oil camellia seeds in this study; Table S9: Novel miRNAs in tea oil camellia seeds in this study; Table S10: Significantly differentially expressed miRNAs in seeds of the same development stage between the two cultivars of 'M3' and 'M8' of tea oil camellia; Table S11: Predicted targets for known and novel miRNAs and their functional annotations in tea 
oil camellia seeds; Table S12: Analysis of the target genes by significant GO enrichment; Table S13: Analysis of the target genes by significant KEGG enrichment; Table S14: KEGG enrichment analysis of differentially expressed miRNAs and their target genes related to lipid metabolism; Table S15: Function of target genes involved in seed traits in tea oil camellia in this study; Figure S1: Pearson correlation between samples; Figure S2: Secondary structures of the five most abundant novel miRNA precursors in tea oil camellia; Figure S3: Bar plot of differentially expressed miRNAs among different groups. Note: the number of upregulated (red) and downregulated (green) miRNAs.

Author Contributions: B.W. performed the experiments of this study and wrote the manuscript; C.R. conceived this study and designed the experiments of this study; A.H.S. helped designed the experiments of this study and revised the manuscript; D.L., H.L., J.D., J.L. and W.D. collected the samples of this study. All authors have read and agreed to the published version of the manuscript.

Funding: This work was supported by the Science and Technology Support Project of Guizhou Province (CN) (Qiankehezhicheng [2022] Zhongdian 017, Qiankehezhicheng [2019] 2404), the Special Forestry industry Science \& Technology R\&D project for Guizhou Rural Industrial Revolution (Telinyan2020-07), and the agricultural special projects of Tongren City (Tongshikeyan [2021]).

Institutional Review Board Statement: Not applicable.

Informed Consent Statement: Not applicable.

Data Availability Statement: Not applicable.

Acknowledgments: The authors thank Sihei Liu and He Huang for their assistance in collecting plant materials.

Conflicts of Interest: The authors declare that they have no competing interests.

\begin{abstract}
Abbreviations
ACC: Acetyl-CoA carboxylase; ACS1: Acyl-CoA synthetase-1; AFB2: Auxin signaling F-box 2; AP2: Apetala2; ARF: Auxin response factor; BAG7: BCL2-associated athanogene 7; CNR: Colourless nonripening; EF1 $\alpha$ : Elongation factor $1 \alpha$; COL13: Collagen type XIII; CPR30: Cytochrome P450 reductase 30; DGAT: Diacylglycerol O-acyltransferase; ERF15: Ethylene-responsive factor 15; fabG: 3-ketoacylacyl carrier protein reductase; FAD2: Fatty acid desaturase 2; FATB1: Fatty acyl-ACP thioesterase B-type 1; GAPN: Glyceraldehyde-3-phosphate dehydrogenase; GPD1: Glycerol-3-phosphate dehydrogenase; GRF: Growth-regulating factor; KAR: 3-ketoacyl-ACP reductase; KAS: 3-ketoacyl-ACP synthase; Mcat: Malonyl-CoA-acyl carrier protein transacylase; MED: Mediator Complex Subunit; NRT1.7: Nitrate nitrogen transporter 1.7; PAB2: Polyadenylate-binding protein 2; S-ACP-DES6: Secretory acid phosphatase desaturase 6; SAD: Stearoyl-acyl carrier protein desaturase; SBP2: Spherical body protein 2; SCL: Stem cell leukemia; SPL: Squamosa promoter binding protein-like; SPL10: SBP-like Protein10; T1R1: Taste receptor T1R1; SAD: stearoyl-ACP desaturase.
\end{abstract}

\title{
References
}

1. Ohlrogge, J.B. Design of new plant products: Engineering of fatty acid metabolism. Plant Physiol. 1999, 104, 821-826. [CrossRef]

2. Li, H.; Zhou, G.Y.; Zhang, H.Y.; Liu, J.A. Research progress on the health function of tea oil. J. Med. Plants Res. $2011,5,485-489$.

3. Lee, C.P.; Yen, G.C. Antioxidant activity and bioactive compounds of tea seed (Camellia oleifera Abel.) oil. J. Agric. Food Chem. 2006, 54, 779-784. [CrossRef]

4. Yu, Y.; Ren, S.; Tan, K. Study on climatic regionalization and layer and belt distribution of oiltea Camellia quality in China. J. Nat. Res. 1999, 14, 123-127.

5. Chen, J.; Yang, X.; Huang, X.; Duan, S.; Long, C.; Chen, J.; Rong, J. Leaf transcriptome analysis of a subtropical evergreen broadleaf plant, wild oil-tea camellia (Camellia oleifera), revealing candidate genes for cold acclimation. BMC Genom. 2017, 18, 211. [CrossRef]

6. Bartel, D.P. MicroRNAs: Genomics, biogenesis, mechanism, and function. Cell 2004, 116, 281-297. [CrossRef]

7. Wang, H.; Zhang, X.; Liu, J.; Kiba, T.; Woo, J.; Ojo, T.; Hafner, M.; Tuschl, T.; Chua, N.H.; Wang, X.J. Deep sequencing of small RNAs specifically associated with Arabidopsis AGO1 and AGO4 uncovers new AGO functions. Plant J. 2011, 67, $292-304$. [CrossRef] [PubMed]

8. Liu, Y.; Wang, L.; Chen, D.; Wu, X.M.; Huang, D.; Chen, L.L.; Li, L.; Deng, X.X.; Xu, Q. Genome-wide comparison of microRNAs and their targeted transcripts among leaf, flower and fruit of sweet orange. BMC Genom. 2014, 15, 695. [CrossRef] 
9. Yin, D.D.; Li, S.S.; Shu, Q.Y.; Gu, Z.Y.; Wang, L.S. Identification of microRNAs and long non-coding RNAs involved in fatty acid biosynthesis in tree peony seeds. Gene 2018, 666, 72-82. [CrossRef]

10. Zhao, X.; Yang, G.; Liu, X.; Yu, Z.; Peng, S. Integrated analysis of seed microrna and mrna transcriptome reveals important functional genes and microrna-targets in the process of walnut (Juglans regia) seed oil accumulation. Int. J. Mol. Sci. 2020, 21, 9093. [CrossRef]

11. Wang, L.; Ruan, C.J.; Bao, A.; Li, H. Small RNA proling for identification of microRNAs involved in regulation of seed development and lipid biosynthesis in yellowhorn. BMC Plant Biol. 2021, 21, 464. [CrossRef] [PubMed]

12. Cao, S.; Zhu, Q.H.; Shen, W.; Jiao, X.; Zhao, X.; Wang, M.B.; Liu, L.; Singh, S.P.; Liu, Q. Comparative profiling of microRNA expression in developing seeds of high linoleic and high oleic safflower (Carthamus tinctorius L.) plants. Front. Plant Sci. 2013, 4 489. [CrossRef] [PubMed]

13. Niu, J.; Wang, J.; An, J.; Liu, L.; Lin, Z.; Wang, R.; Wang, L.; Ma, C.; Shi, L.; Lin, S. Integrated mRNA and miRNA transcriptome reveal a cross-talk between developing response and hormone signaling for the seed kernels of Siberian apricot. Sci. Rep. 2016, 6, 35675. [CrossRef]

14. Mutum, R.D.; Kumar, S.; Balyan, S.; Kansal, S.; Mathur, S.; Raghuvanshi, S. Identification of novel miRNAs from drought tolerant rice variety Nagina 22. Sci. Rep. 2016, 6, 30786. [CrossRef]

15. Subramanian, S.; Steer, C.J. MicroRNAs as gatekeepers of apoptosis. J. Cell. Physiol. 2010, 223, 289-298. [CrossRef]

16. Cuperus, J.T.; Fahlgren, N.; Carrington, J.C. Evolution and functional diversification of miRNA genes. Plant Cell 2011, $23,431-442$. [CrossRef] [PubMed]

17. Griffiths-Jones, S.; Saini, H.K.; Dongen, S.V.; Enright, A.J. miRbase: Tools for microRNA genomics. Nucl. Acids Res. 2008, 36, D154-D158. [CrossRef]

18. Song, Q.X.; Liu, Y.F.; Hu, X.Y.; Zhang, W.K.; Ma, B.; Chen, S.Y.; Zhang, J.S. Identification of miRNAs and their target genes in developing soybean seeds by deep sequencing. BMC Plant Biol. 2011, 11, 5. [CrossRef]

19. Reyes, J.L.; Chua, N.H. ABA induction of miR159 controls transcript levels of two MYB factors during Arabidopsis seed germination. Plant J. 2007, 49, 592-606. [CrossRef] [PubMed]

20. Körbes, A.P.; Machado, R.D.; Guzman, F.; Almerão, M.P.; de Oliveira, L.F.; Loss-Morais, G.; Turchetto-Zolet, A.C.; Cagliari, A.; dos Santos Maraschin, F.; Margis-Pinheiro, M.; et al. Identifying conserved and novel microRNAs in developing seeds of Brassica napus using deep sequencing. PLoS ONE 2012, 7, e50663. [CrossRef] [PubMed]

21. Peng, T.; Sun, H.; Qiao, M.; Zhao, Y.; Du, Y. Differentially expressed microRNA cohorts in seed development may contribute to poor grain filling of inferior spikelets in rice. BMC Plant Biol. 2014, 14, 196. [CrossRef] [PubMed]

22. Jin, Q.; Xue, Z.; Dong, C.; Wang, Y.; Chu, L.; Xu, Y. Identification and characterization of microRNAs from tree peony (Paeonia ostii) and their response to copper stress. PLOS ONE 2015, 10, e0117584. [CrossRef]

23. Li, D.; Wang, L.; Liu, X.; Cui, D.; Chen, T.; Zhang, H. Deep sequencing of maize small RNAs reveals a diverse set of microRNA in dry and imbibed seeds. PLoS ONE 2013, 8, e55107. [CrossRef]

24. Ding, J.; Ruan, C.; Guan, Y. Identification of microRNAs involved in lipid biosynthesis and seed size in developing sea buckthorn seeds using high-throughput sequencing. Sci. Rep. 2018, 8, 4022. [CrossRef] [PubMed]

25. Belide, S.; Petrie, J.R.; Shrestha, P.; Singh, S.P. Modification of seed oil composition in Arabidopsis by artificial microRNA-mediated gene silencing. Front. Plant Sci. 2012, 3, 168. [CrossRef] [PubMed]

26. Na, G.N.; Mu, X.; Grabowski, P.; Schmutz, J.; Lu, C. Enhancing microrna167a expression in seed decreases the $\alpha$-linolenic acid content and increases seed size in Camelina sativa. Plant J. 2019, 98, 346-358. [CrossRef] [PubMed]

27. Tan, H.; Yang, X.; Zhang, F.; Zheng, X.; Qu, C.; Mu, J.; Fu, F.; Li, J.; Guan, R.; Zhang, H.; et al. Enhanced seed oil production in Canola by conditional expression of Brassica napus LEAFY COTYLEDON1 and LEC1-LIKE in developing seeds. Plant Physiol. 2011, 156, 1577-1588. [CrossRef]

28. Kwong, R.W.; Bui, A.Q.; Lee, H.; Kwong, L.W.; Fischer, R.L.; Goldberg, R.B.; Harada, J.J. LEAFY COTYLEDON1-LIKE defines a class of regulators essential for embryo development. Plant Cell 2003, 15, 5-18. [CrossRef] [PubMed]

29. Gupta, M.; Bhaskar, P.B.; Sriram, S.; Wang, P.H. Integration of omics approaches to understand oil/protein content during seed development in oilseed crops. Plant Cell Rep. 2016, 36, 637-652. [CrossRef]

30. Khemka, N.; Rajkumar, M.S.; Garg, R.; Jain, M. Genome-wide profiling of miRNAs during seed development reveals their functional relevance in seed size/weight determination in chickpea. Plant Direct 2021, 5, e00299. [CrossRef] [PubMed]

31. Parreira, J.R.; Cappuccio, M.; Balestrazzi, A.; Fevereiro, P.; de Sousa Araújo, S. Micrornas expression dynamics reveal posttranscriptional mechanisms regulating seed development in Phaseolus vulgaris L. Hortic. Res. 2021, 8, 18. [CrossRef]

32. Liu, P.P.; Montgomery, T.A.; Fahlgren, N.; Kasschau, K.D.; Carrington, J.C. Repression of AUXIN RESPONSE FACTOR10 by microRNA160 is critical for seed germination and post-germination stages. Plant J. 2010, 52, 133-146. [CrossRef]

33. Nodine, M.D.; Bartel, D.P. MicroRNAs prevent precocious gene expression and enable pattern formation during plant embryogenesis. Gene Dev. 2010, 24, 2678-2692. [CrossRef]

34. Allen, R.S.; Li, J.; Stahle, M.I.; Aurelie, D.; Gubler, F.; Millar, A.A. Genetic analysis reveals functional redundancy and the major target genes of the Arabidopsis miR159 family. Proc. Natl. Acad. Sci. USA 2007, 104, 16371-16376. [CrossRef] [PubMed]

35. Li, J.B.; Ding, J.; Yu, R.; Li, H.; Ruan, C.J. Identification and expression analysis of critical microrna-transcription factor regulatory modules related to seed development and oil accumulation in developing Hippophae rhamnoides seeds. Ind. Crops Prod. 2019, 137, 33-42. [CrossRef] 
36. Liu, J.; Luo, Q.; Zhang, X.; Zhang, Q.; Cheng, Y. Identification of vital candidate microRNA/mRNA pairs regulating ovule development using high-throughput sequencing in hazel. BMC Dev. Biol. 2020, 20, 13. [CrossRef]

37. Harikrishna, K.; Othman, R.Y.; Hoon, L.S.; Harikrishna, J.A. Computational prediction of microRNAs from oil palm (Elaeis guineensis Jacq.) expressed sequence tags. Asia-Pac. J. Mol. Biol. Biotechnol. 2007, 15, 107-113.

38. Donaire, L.L.; Pedrola, L.L.; Rosa, R.D.L.; Liave, C. High-throughput sequencing of RNA silencing-associated small RNAs in olive (Olea europaea L.). PLoS ONE 2011, 6, e27916. [CrossRef] [PubMed]

39. Ding, J.; Ruan, C.J.; Du, W.; Guan, Y. RNA-seq data reveals a coordinated regulation mechanism of multigenes involved in the high accumulation of palmitoleic acid and oil in sea buckthorn berry pulp. BMC Plant Biol. 2019, 19, 207. [CrossRef] [PubMed]

40. Wu, B.; Ruan, C.J.; Ding, J.; Du, W.; Li, J.B.; Yan, R.; Liu, L.R. Coordinated expression of source and sink genes involved in lipid biosynthesis and accumulation during camellia oleifera seed development. Acta Bot. Boreal.-Occident. Sin. 2017, 37, 2483-2488, (In Chinese with an English abstract).

41. Wu, B.; Ruan, C.J.; Han, P.; Ruan, D.; Xiong, C.W.; Ding, J.; Liu, S.H. Comparative transcriptomic analysis of high- and low-oil Camellia oleifera reveals a coordinated mechanism for the regulation of upstream and downstream multigenes for high oleic acid accumulation. 3 Biotech 2019, 9, 257. [CrossRef]

42. Han, X.; Yin, H.; Song, X.; Zhang, Y.; Liu, M.; Sang, J.; Jiang, J.; Li, J.; Zhuo, R. Integration of small RNAs, degradome and transcriptome sequencing in hyperaccumulator Sedum alfredii uncovers a complex regulatory network and provides insights into cadmium phytoremediation. Plant Biotechnol. J. 2016, 14, 1470-1483. [CrossRef]

43. miRBase 20.0. Available online: $\mathrm{ftp}: / /$ mirbase.org/pub/mirbase/CURRENT/ (accessed on 20 July 2015).

44. Ambady, S.; Wu, Z.Y.; Dominko, T. Identifification of novel microRNAs in Xenopus laevis Metaphase II arrested eggs. Genesis 2012, 50, 286-299. [CrossRef]

45. Li, X.; Shahid, M.Q.; Wu, J.W.; Wang, L.; Liu, X.D.; Lu, Y.G. Comparative small RNA analysis of pollen development in autotetraploid and diploid rice. Int. J. Mol. Sci. 2016, 17, 499. [CrossRef] [PubMed]

46. Cer, R.Z.; Herrera-Galeano, J.E.; Anderson, J.J.; Bishop-Lilly, K.A.; Mokashi, V.P. miRNA Temporal Analyzer (mirnaTA): A bioinformatics tool for identifying differentially expressed microRNAs in temporal studies using normal quantile transformation. GigaScience 2014, 3, 20. [CrossRef] [PubMed]

47. Fahlgren, N.; Carrington, J.C. miRNA target prediction in plants. Methods Mol. Biol. 2010, 592, 51-57. [PubMed]

48. Schwab, R.; Palatnik, J.F.; Riester, M.; Schommer, C.; Schmid, M.; Weigel, D. Specific effects of microRNAs on the plant transcriptome. Dev. Cell 2005, 8, 517-527. [CrossRef] [PubMed]

49. Schmittgen, T.D.; Livak, K.J. Analyzing real-time PCR data by the comparative C(T) method. Nat. Protoc. 2008, 3, 1101-1108. [CrossRef] [PubMed]

50. Vidal, E.A.; Araus, V.; Lu, C.; Parry, G.; Green, P.J.; Coruzzi, G.M.; Gutierrez, R.A. Nitrate-responsive miR393/AFB3 regulatory module controls root system architecture in Arabidopsis thaliana. Proc. Natl. Acad. Sci. USA 2010, 107, 4477-4482. [CrossRef] [PubMed]

51. Lakehal, A.; Chaabouni, S.; Cavel, E.; Hir, R.L.; Bellini, C. A molecular framework for the control of adventitious rooting by the TIR1/AFB2-Aux/IAA-Dependent auxin signaling in Arabidopsis. Mol. Plant 2019, 12, 1499-1514. [CrossRef]

52. Shimono, Y.; Zabala, M.; Cho, R.W.; Lobo, N.; Dalerba, P.; Qian, D. Downregulation of miRNA-200c links breast cancer stem cells with normal stem cells. Cell 2009, 138, 592-603. [CrossRef]

53. Agatheeswaran, S.; Pattnayak, N.C.; Chakraborty, S. Identification and functional characterization of the miRNA-gene regulatory network in chronic myeloid leukemia lineage negative cells. Sci. Rep. 2016, 6, 32493. [CrossRef] [PubMed]

54. Kumari, B.; Jain, P.; Das, S.; Ghosal, S.; Hazra, B.; Trivedi, A.C.; Basu, A.; Chakrabarti, J.; Vrati, S.; Banerjee, J. Dynamic changes in global microRNAome and transcriptome reveal complex miRNA-mRNA regulated host response to Japanese encephalitis Virus in microglial cells. Sci. Rep. 2016, 6, 20263. [CrossRef]

55. Qian, P.; Jiang, T.; Wang, X.; Song, F.; Shen, X. Bmo-miR-275 down-regulates expression of Bombyx mori sericin gene 2 in vitro. PLoS ONE 2018, 13, e0190464. [CrossRef] [PubMed]

56. Dai, X.; Zhao, P.X. psRNATarget: A plant small RNA target analysis server. Nucleic Acids Res. 2011, 39, 155-159. [CrossRef]

57. Bonnet, E.; Wuyts, J.; Rouzé, P.; van de Peer, Y. Detection of 91 potential conserved plant microRNAs in Arabidopsis thaliana and Oryza sativa identifies important target genes. Proc. Natl. Acad. Sci. USA 2004, 101, 11511-11516. [CrossRef] [PubMed]

58. Baker, C.C.; Sieber, P.; Wellmer, F.; Meyerowitz, E.M. The early extra petals1 mutant uncovers a role for microRNA miR164c in regulating petal number in Arabidopsis. Curr. Biol. 2005, 15, 303-315. [CrossRef]

59. Weber, M.J. New human and mouse microRNA genes found by homology search. FEBS J. 2010, 272, 59-73. [CrossRef]

60. Zhang, B.; Pan, X.; Cannon, C.H.; Cobb, G.P.; Anderson, T.A. Conservation and divergence of plant microRNA genes. Plant J. 2010, 46, 243-259. [CrossRef]

61. Zhao, Y.T.; Wang, M.; Fu, S.X.; Yang, W.C.; Qi, C.K.; Wang, X.J. Small RNA profiling in two Brassica napus cultivars identifies microRNAs with oil production- and development-correlated expression and new small RNA classes. Plant Physiol. 2012, 158, 813-823. [CrossRef]

62. Lai, C.Y.; Cronan, J.E. Isotation and characterization of $\beta$-ketoacyl-acyl carrier protein reductase (fabG) mutants of Escherichia coli and Salmonella enterica serovar Tyhimurium. J. Bacteriol. 2004, 186, 1869-1878. [CrossRef] [PubMed]

63. Huang, D.; Koh, C.; Feurtado, J.A.; Tsang, E.W.; Cutler, A.J. MicroRNAs and their putative targets in Brassica napus seed maturation. BMC Genom. 2013, 14, 140. [CrossRef] 
64. Wang, B.; Tan, X.; Jiang, J.; Zhang, L. Molecular cloning and expression of two genes encoding accase subunits of Camellia oleifera (Theaceae). Pak. J. Bot. 2018, 50, 103-110.

65. Andre, C.; Haslam, R.P.; Shanklin, J. Feedback regulation of plastidic acetyl-CoA carboxylase by 18:1-acyl carrier protein in Brassica napus. Proc. Natl. Acad. Sci. USA 2012, 109, 10107-10112. [CrossRef]

66. Nakkaew, A.; Chotigeat, W.; Eksomtramage, T.; Phongdara, A. Cloning and expression of a plastid-encoded subunit, betacarboxyltransferase gene (accD) and a nuclear-encoded subunit, biotin carboxylase of acetyl-CoA carboxylase from oil palm (Elaeis guineensis Jacq.). Plant Sci. 2008, 175, 497-504. [CrossRef]

67. Lee, B.; Shin, S.E.; Choi, G.G.; Jeong, B.; Park, M.S.; Yang, J.W. Effects of Overexpression of GAPDH on Growth and Lipid Accumulation in Chlamydomonas reinhardtii. 2013. Available online: https:/ / koasas.kaist.ac.kr/handle/10203/188091 (accessed on 23 December 2021).

68. Nofiani, R.; Philmus, B.; Nindita, Y.; Mahmud, T. 3-ketoacyl-acp synthase (kas) iii homologues and their roles in natural product biosynthesis. MedChemComm 2019, 10, 1517-1530. [CrossRef]

69. Sang, Y.S.; Sang, H.K.; Velusamy, V.; Lee, Y.M. Comparative gene expression analysis in a highly anthocyanin pigmented mutant of colorless chrysanthemum. Mol. Biol. Rep. 2013, 40, 5177-5189. [CrossRef]

70. Heath, R.J.; Su, N.; Murphy, C.K.; Rock, C.O. The Enoyl-[acyl-carrier-protein] reductases FabI and FabL from Bacillus subtilis. J. Biol. Chem. 2000, 275, 40128-40133. [CrossRef] [PubMed]

71. Yasuno, R.; von Wettstein-Knowles, P.; Wada, H. Identification and molecular characterization of the beta-Ketoacyl-[Acyl Carrier Protein] synthase component of the Arabidopsis mitochondrial fatty acid synthase. J. Biol. Chem. 2004, 279, 8242-8251. [CrossRef] [PubMed]

72. Zhang, D.Q.; Tan, X.F.; Hu, F.M. The cDNA cloning and characteristic of stearoyl-ACP desaturase gene of Camellia oleifera. Acta Hortic. 2008, 769, 55-61. [CrossRef]

73. Kachroo, A.; Shanklin, J.; Whittle, E.; Lapchyk, L.; Hildebrand, D.; Kachroo, P. The Arabidopsis stearoyl-acyl carrier proteindesaturase family and the contribution of leaf isoforms to oleic acid synthesis. Plant Mol. Biol. 2007, 63, 257-271. [CrossRef] [PubMed]

74. Dörmann, P.; Voelker, T.A.; Ohlrogge, J.B. Accumulation of palmitate in Arabidopsis mediated by the acyl-acyl carrier protein thioesterase FATB1. Plant Physiol. 2000, 123, 637-643. [CrossRef] [PubMed]

75. Xu, R.; Li, Y. Control of final organ size by mediator complex subunit 25 in Arabidopsis thaliana. Development 2011, 22, 4545-4554. [CrossRef] [PubMed]

76. Guo, M.; Simmons, C.R. Cell number counts-The fw2.2 and CNR genes and implications for controlling plant fruit and organ size. Plant Sci. 2011, 181, 1-7. [CrossRef]

77. Wu, G.; Park, M.Y.; Conway, S.R.; Wang, J.W.; Weigel, D.; Poethig, R.S. The sequential action of miR156 and miR172 regulates developmental timing in Arabidopsis. Cell 2009, 138, 750-759. [CrossRef] [PubMed]

78. Abdalla, A.H.S. Characterization of a MYB Gene from Tomato (Solanum lycopersicum) Involved in Fruit Development. Master's Thesis, Universitat Politècnica de València, Valencia, Spain, 2013.

79. Li, D.; Jin, C.; Duan, S.; Zhu, Y.; Qi, S.; Liu, K.; Gao, C.; Ma, H.; Zhang, M.; Liao, Y.; et al. MYB89 transcription factor represses seed oil accumulation. Plant Physiol. 2017, 173, 1211-1225. [CrossRef] [PubMed]

80. Han, W.; Mao, Y.; Yang, J.; He, Y. TCP24 modulates secondary cell wall thickening and anther endothecium development. Front. Plant Sci. 2015, 6, 436. [CrossRef]

81. Wu, J.Y.; Li, C.Q.; Li, J.G.J. Isolation of acs1 gene and the relationship between its expression and fruitlet abscission in litchi. J. Fruit Sci. 2017, 34, 817-827.

82. Yu, Z.; Ma, X.; Zeng, B.; Wang, J.; Aymt, A. Research progress on sbp1 gene of plant gametophytic self-incompatibility. Mol. Plant Breed. 2019, 17, 5285-5290, (In Chinese with an English abstract).

83. Freitas, R.L.; Carvalho, C.M.; Fietto, L.G.; Loureiro, M.E.; Almeida, A.M.; Fontes, E. Distinct repressing modules on the distal region of the sbp2 promoter contribute to its vascular tissue-specific expression in different vegetative organs. Plant Mol. Biol. 2007, 65, 603-614. [CrossRef]

84. Sunkar, R.; Zhu, J.-K. Novel and stress-regulated microRNAs and other small RNAs from Arabidopsis. Plant Cell 2004, 16, 2001-2019. [CrossRef] [PubMed]

85. Huh, S.U.; Kim, M.J.; Paek, K.H. Arabidopsis Pumilio protein APUM5 suppresses Cucumber mosaic virus infection via direct binding of viral RNAs. Proc. Natl. Acad. Sci. USA 2013, 110, 779-784. [CrossRef]

86. Wei, R.M.; Xie, L.L.; Ouyang, X.; Zhang, Y.L.; Dai, Z.Z.; Liu, F. The Structure and Functional Characteristics of Plant BAG Protein Family. Hunan Agric. Sci. 2016, 9, 115-120, (in Chinese with an English abstract).

87. Gou, M.; Nan, S.; Zheng, J.; Huai, J.; Wang, G. An f-box gene, cpr30, functions as a negative regulator of the defense response in Arabidopsis. Plant J. 2010, 60, 757-770. [CrossRef] [PubMed]

88. Tripathi, S.; Srivastava, Y.; Sangwan, R.S.; Sangwan, N.S. Mining and functional analysis of ap2/erf gene in. Sci. Rep. 2020, 10, 4877. [CrossRef] [PubMed]

89. Ouyang, J. The Mechanism of MYB4 Gene Involved in the Response of Drought Stress in Plants. Master's Thesis, Hefei University of Technology, Hefei, China, 2019.

90. Pan, Z.Y. Mapping of QTLs Controlling Seed Low Temperature Germinability and Fine Mapping and Transcriptome Analysis of qLTGR3-1 in Rice. Master's Thesis, Huazhong Agricultural University, Wuhan, China, 2018. 
91. Peragine, A.; Yoshikawa, M.; Wu, G.; Albrecht, H.L.; Poethig, R.S. SGS3 and SGS2/SDE1/RDR6 are required for juvenile development and the production of trans-acting siRNAs in Arabidopsis. Genes Dev. 2004, 18, 2368-2379. [CrossRef] [PubMed]

92. Wang, S.; Li, S.; Liu, Q. The OsSPL16-GW7 regulatory module determines grain shape and simultaneously improves rice yield and grain quality. Nat. Genet. 2014, 47, 949-954. [CrossRef] [PubMed]

93. Zhao, L.; Zhang, W.; Yang, Y. The Arabidopsis NLP7 gene regulates nitrate signaling via NRT1.1-dependent pathway in the presence of ammonium. Sci. Rep. 2018, 8, 1487. [CrossRef] 